\title{
Kähler-Einstein metrics of negative Ricci curvature on general quasi-projective manifolds
}

\author{
DAMIN WU
}

\begin{abstract}
In this paper, we give sufficient and necessary conditions for the existence of a Kähler-Einstein metric on a quasi-projective manifold of finite volume, bounded Riemannian sectional curvature and Poincaré growth near the boundary divisor. These conditions are obtained by solving a degenerate Monge-Ampère equation and deriving the asymptotics of the solution.
\end{abstract}

\section{Introduction}

Let $M$ be a quasi-projective manifold which can be compactified by adding a divisor $D$ with simple normal crossings, which means that $D=\sum_{i=1}^{p} D_{i}$, where the irreducible components $D_{i}$ are smooth and intersect transversely. Under certain positivity conditions of the adjoint bundle over the compactification, the existence of a complete Kähler-Einstein metric on the quasi-projective manifold was first addressed by Yau (see, for example, [29, p. 166]), right after his resolution of the Calabi conjecture [28]. This program has been followed by many authors, for example, [1, 5, 8, 22, 24], and [31].

In fact, notice that the second part of [28] is essentially devoted to construct Kähler-Einstein metrics on algebraic manifolds of general type. In general such a metric has singularities. In a sense, Yau's motivation for this program may be viewed as to understand these singularities in many important cases from the differential-geometric point of view. This appears in his later papers joint with Cheng [8], and with Tian [22]. In the last paper, they proved the following result.

Let

$$
K_{\bar{M}}+\sum_{i=1}^{q}\left[D_{i}\right]+\sum_{j=q+1}^{p} \frac{m_{j}-1}{m_{j}}\left[D_{j}\right]
$$

be big, nef, and ample modulo $\sum_{i=1}^{q} D_{i}$, where $K_{\bar{M}}$ denotes the canonical bundle over $\bar{M},\left[D_{i}\right]$ is the line bundle induced from $D_{i}, m_{j} \in \mathbb{N}$ and $m_{j} \geq 2$. Then there exists a unique (almost) complete Kähler-Einstein metric with 
negative Ricci curvature on $M$. (See Section 7 here for definition of almost completeness. The completeness is later settled by Yau [31, p. 474].) As a consequence, the logarithmic version of Miyaoka-Yau inequality is established on $M$ and a numerical characterization of ball quotients is given (see [22, p. 626]).

Note that the condition of (1.1) is equivalent to the following (see [22, p. 612]): There exist $\mu_{i} \in \mathbb{Q}, \mu \in(0,1]$, for $i=1, \ldots, q$, and $m_{j} \in \mathbb{N}, m_{j} \geq 2$ such that

$$
K_{\bar{M}}+\sum_{i=1}^{q} \mu_{i}\left[D_{i}\right]+\sum_{j=q+1}^{p} \frac{m_{j}-1}{m_{j}}\left[D_{j}\right]>0
$$

on $\bar{M}$ and

$$
K_{\bar{M}}+\sum_{i=1}^{q}\left[D_{i}\right]+\sum_{j=q+1}^{p} \frac{m_{j}-1}{m_{j}}\left[D_{j}\right] \quad \text { is nef. }
$$

The positivity condition (1.2) assures that, on $M$, there exists a natural complete Kähler metric, which has Poincaré growth near the divisor $\sum_{i=1}^{q} D_{i}$. This background metric can be deformed to a complete Kähler-Einstein metric by solving a degenerate Monge-Ampère equation on $M$. (For the condition corresponding to a nondegenerate Monge-Ampère equation, the existence has also been studied from the viewpoint of Ricci flow, see Chau [4], which is parallel to Cao's work [3] on the compact case.)

In [22], a modified continuity method is introduced to solve the equation. The nef condition (1.3) is imposed so that the deformation of metrics would not be out of control until $t=1$. But then it is nontrivial to show the completeness of the resulting metric. The key observation in [31] is that the Kähler-Einstein metric can dominate a Poincaré-type metric which has negative holomorphic curvature at least in the normal direction. This is proved by the argument in Yau's Schwarz lemma [30] together with the property of almost completeness.

In this paper, we would like to consider a general positivity assumption, under which $M$ admits a Kähler-Einstein metric of a negative Ricci curvature. Under the assumption, it turns out that the information coming from the boundary divisor is crucial for the Kähler-Einstein geometry over the whole quasi-projective manifold. Indeed, let us relax the positivity 
(1.2) to the following condition: There exist real numbers $\alpha_{i} \in[-1,+\infty)$, $i=1, \ldots, p$, so that

$$
K_{\bar{M}}-\sum_{i=1}^{p} \alpha_{i}\left[D_{i}\right]>0
$$

on $\bar{M}$. This assumption is pretty general for the existence of a KählerEinstein metric with negative Ricci curvature on $M$. In fact, if we allow some coefficient $\alpha_{k}<-1$, then it is possible that there is no Kähler-Einstein metric of negative Ricci curvature on $M$. For instance, let $\bar{M}=\mathbb{C} P^{n}$ and $D$ be a smooth hypersurface of degree $n+1$. Then (1.4) holds for any $\alpha<-1$. But by [23] there exists a complete Kähler-Ricci flat metric on $M$. Thus, $M$ does not admit any Kähler-Einstein metric of negative Ricci curvature, in view of Yau's Schwarz lemma.

Under an assumption like (1.4), it is standard to construct a complete Kähler metric $\omega$ on $M$ with Poincaré growth near $D$. Furthermore, $\omega$ has bounded sectional curvature, and indeed, has bounded geometry in the sense of Cheng-Yau (see, for example, [26, p. 800-802]). Let $\mathcal{R}(M)$ be the ChengYau's Hölder ring (see Section 2 for definition). Our first result is on the existence of a negative Kähler-Einstein metric on $M$, which is parallel to part of Yau's work on the compact degenerate case [28, p. 364-389].

Theorem 1.1. Under (1.4), there exists a Kähler-Einstein metric $\omega_{\mathrm{KE}}$ on $M$ of finite volume and negative Ricci curvature, satisfying that

$$
C^{-1} \prod_{i=1}^{p}\left|s_{i}\right|^{2 \Lambda} \omega<\omega_{\mathrm{KE}}<C \prod_{i=1}^{p}\left|s_{i}\right|^{-2 \Lambda} \omega,
$$

where $s_{i}$ is the holomorphic defining section of $D_{i},|\cdot|$ is a metric on $\left[D_{i}\right]$, $i=1, \ldots, p$, and $C$ and $\Lambda$ are positive constants.

Here, the finite volume property follows directly from Yau's Schwarz lemma ([30, p. 202]); see also Lemma 3.4 of this paper. The proof of existence amounts to solving a degenerate Monge-Ampère equation, similar to the previous work. However, without any extra assumption like (1.3), the continuity method will break down. So instead we use an approximation method modified from the second part of Yau's celebrated paper [28, pp. 364-389]. It is unclear whether such a Kähler-Einstein metric is complete or not in the general setting, which is the main drawback of the theorem.

On the other hand, for a Kähler-Einstein metric of negative Ricci curvature, if complete, then it must be unique, and depends only on the complex 
structure. (This fact is also an immediate consequence of Yau's Schwarz lemma [30].) Hence, the complete metric imposes certain restrictions on the complex structure of the manifold. Besides, from the differential-geometric viewpoint of compactification (see, for example, [18,32, p. 13]), it would be helpful to compare the Kähler-Einstein metric with the background metric $\omega$, which has bounded curvature. The following theorem can be viewed as the main result of this paper.

Theorem 1.2. The following results are based on the assumption of (1.4).

(1) If $K_{\bar{M}}+[D]$ restricted to each irreducible component of $D$ is positive, then there exists a Kähler-Einstein metric $\omega_{\mathrm{KE}}$ of negative Ricci curvature which is uniformly equivalent ${ }^{1}$ to $\omega$, i.e., there exists some constant $C>0$ such that

$$
C^{-1} \omega<\omega_{\mathrm{KE}}<C \omega .
$$

As a consequence, the Kähler-Einstein metric is complete, has finite volume and bounded sectional curvature.

(2) If there exists a Kähler-Einstein metric of negative Ricci curvature $\omega_{\mathrm{KE}}$ on $M$ which dominates $\omega$, namely, $\omega_{\mathrm{KE}}>C \omega$, for some constant $C>0$, then $\omega_{\mathrm{KE}}$ is uniformly equivalent to $\omega$, and is given by

$$
\omega_{\mathrm{KE}}=\omega-\sum_{i=1}^{p}\left(\alpha_{i}+1\right) d d^{c} \log \left|s_{i}\right|^{2}+d d^{c} v,
$$

where $v \in \mathcal{R}(M)$ and $\alpha_{i}$ are the coefficients in (1.4). As a consequence, $\omega_{\mathrm{KE}}$ has bounded sectional curvature, and

$$
\omega_{\mathrm{KE}}^{n}=\frac{e^{v} V}{\prod_{i=1}^{p}\left|s_{i}\right|^{2}\left(\log \left|s_{i}\right|^{2}\right)^{2}},
$$

where $V$ is a volume form on $\bar{M}$.

We remark that, unlike the nefness of $K_{\bar{M}}+[D]$, under

$$
\left.\left(K_{\bar{M}}+[D]\right)\right|_{D_{i}}>0, \quad i=1, \ldots, p
$$

the resulting Käher-Einstein metric on $M$ is uniformly equivalent to the background metric. This endows the new Käher-Einstein metric with all the merits of $\omega$, especially the bounded sectional curvature property.

\footnotetext{
${ }^{1}$ This may also be called as quasi-isometric in the literature.
} 
As far as (2) in Theorem 1.2 is concerned, the coefficients $\alpha_{i}$ in (1.4) are allowed to be any real numbers. We derive (2) by studying the asymptotics of the unbounded solution of the degenerate Monge-Ampère equation. We introduce the Cheng-Yau's Hölder spaces of negative weight to characterize the unbounded solution. Then we derive the asymptotics by extending the isomorphism theorems developed in [26] to the negative weight spaces. Specifically, we show that the solution has a logarithmic pole of order $\alpha_{i}+1$ along each irreducible component of $D$. See Theorem 5.3 for details and also for certain uniqueness. The idea of using isomorphism theorems originally came from Lee and Melrose [14] in handling the asymptotics in the strictly pseudoconvex domain in $\mathbb{C}^{n}$ (see also, for example, $[7,10]$ for the background.)

Statement (1) in Theorem 1.2 is proved by the continuity method. It is more or less standard that such a proof can be reduced to some a priori estimates near the boundary divisor (see, for example, [22, Theorem 1.1, p. 581]). An important ingredient here is a generalized Saper's lemma on metric extensions, which enables us to obtain the desired estimates near $D$. Then we choose the classical version of the continuity method, together with certain a priori asymptotic treatment, which makes the argument more transparent. By pushing the argument one step further, we have

Theorem 1.3. Under (1.4), if $K_{\bar{M}}+[D]$ restricted to each irreducible component of $D$ is nef, then there exists an (almost) complete Kähler-Einstein metric on $M$.

By using the metric extension lemma, one can again derive the a priori estimates near $D$. Following [22], we use the modified continuity method which has the advantage that the Ricci curvature of the deforming metric always has a lower bound. But one has to pay the cost of nonemptiness. Here, inspired from [19], we use Newton's iteration to overcome the difficulty due to the failure of the usual implicit function theorem.

In Section 2, we recall the setting of bounded geometry, and weighted Cheng-Yau's Hölder spaces. We construct a background Kähler metric adapted to these settings. In Section 3, we collect some well-known and standard techniques, such as Yau's generalized maximum principle and Yau's upper bound lemma. These results are stated and proved in the form we need. In Section 4, a general existence of a Kähler-Einstein metric is proved, by the approximation method. In Section 5, we derive the asymptotics of the solution of the Monge-Ampère equation near $D$. As an application, 
we prove the second part of Theorem 1.2. In Section 6, we prove the first part of Theorem 1.2 by the classical continuity method. Theorem 1.3 is proved in the final section, Section 7.

Throughout this paper, we always identify a Hermitian metric with its associated (1,1)-form. We denote by

$$
\sigma_{i}=-\log \left|s_{i}\right|^{2}, \quad i=1, \ldots, p .
$$

And in fact, we will use both notations interchangeably. For instance, $\sigma_{i}$ is used in Cheng-Yau's Hölder spaces as the weight function, while $-\log \left|s_{i}\right|^{2}$ is used in the estimates of the Monge-Ampère equation. The operator $d^{c}$ is given by

$$
d^{c}=\frac{\sqrt{-1}}{4 \pi}(\bar{\partial}-\partial),
$$

and hence

$$
d d^{c}=\frac{\sqrt{-1}}{2 \pi} \partial \bar{\partial} .
$$

By a Ricci form of a volume form $\Psi$ we mean

$$
\operatorname{Ric}(\Psi)=d d^{c} \log \gamma_{\alpha},
$$

where $\Psi=\gamma_{\alpha} \prod_{i}\left((\sqrt{-1} / 2 \pi) d z_{\alpha}^{i} \wedge d \bar{z}_{\alpha}^{i}\right)$ locally. The Ricci form is in fact globally defined. Notice that $\operatorname{Ric}\left(\omega^{n}\right)$ differs by a negative sign from the usual Ricci curvature of $\omega$.

\section{Bounded Geometry}

Let $\bar{M}$ be a compact complex manifold, and $D=\sum_{i=1}^{p} D_{i}$ an effective divisor, in which the irreducible components $D_{i}$ are smooth and intersect transversely. Let $M=\bar{M} \backslash D$. Suppose that (1.4) holds; namely, there exist real numbers $\alpha_{i} \in[-1,+\infty), i=1, \ldots, p$ such that

$$
K_{\bar{M}}-\sum_{i=1}^{p} \alpha_{i}\left[D_{i}\right]>0
$$

on $\bar{M}$. Then one can construct a complete Kähler metric $\omega$ on $M$ with Poincarè growth near $D$, and has bounded geometry as follows: One can choose a volume form $V$ on $\bar{M}$ and a metric $h_{i}$ on each $\left[D_{i}\right]$ such that the induced metric on $K_{\bar{M}}-\sum_{i=1}^{p} \alpha_{i}\left[D_{i}\right]$ has positive curvature form. Let $s_{i}$ be the holomorphic defining section of $D_{i}$. Then the Ricci form of the 
following volume

$$
\Omega=\prod_{i=1}^{p} \frac{\left|s_{i}\right|^{2 \alpha_{i}}}{\left(\log \left|s_{i}\right|^{2}\right)^{2}} \cdot V
$$

over $M$ is the desired Kähler metric. More precisely, we have

$$
\begin{aligned}
\omega & =\operatorname{Ric}(\Omega) \\
& =\omega_{K}-2 \sum_{i=1}^{p} \sigma_{i}^{-1} \omega_{D_{i}}+2 \sum_{i=1}^{p} \sigma_{i}^{-2} d \sigma_{i} \wedge d^{c} \sigma_{i}
\end{aligned}
$$

over the quasi-projective manifold $M$. Here $\sigma_{i}=-\log \left|s_{i}\right|^{2}$, and

$$
\begin{aligned}
& \omega_{K}=\operatorname{Ric}(V)+\sum_{i=1}^{p} \alpha_{i} d d^{c} \log h_{i} \\
& \omega_{D_{i}}=-d d^{c} \log h_{i}, \quad i=1, \ldots, p
\end{aligned}
$$

represent, respectively, the first Chern classes of $K_{\bar{M}}-\sum_{i=1}^{p} \alpha_{i}\left[D_{i}\right]$ and $\left[D_{i}\right]$, $i=1, \ldots, p$, over $\bar{M}$. Note that we can always rescale the metric on each $\left[D_{i}\right]$ such that

$$
0 \leq\left|s_{i}\right|^{2}<1 / e \text { on } \bar{M}
$$

and hence, we have $\sigma_{i}>1$ on $M$.

Let us recall the setting of bounded geometry (see, for example, $[7,8,22,26])$ over $M$. The idea is to resolve the singularities of the metric near the boundary, by certain nice local holomorphic maps, called quasicoordinate maps. In the case of the quasi-projective manifold, the boundary is the divisor $D=\sum_{i} D_{i}$ with simple normal crossings. For any $p \in D$, there is a neighborhood $U$ of p with $\left(z^{1}, \ldots, z^{n}\right)$ a local coordinate system on $\bar{M}$, such that

$$
D \cap U=\left\{z^{1} \cdots z^{k}=0\right\}, \quad 1 \leq k \leq n
$$

The complement is

$$
U^{*}=U \backslash(D \cap U)=\left(\Delta^{*}\right)^{k} \times \Delta^{n-k},
$$

where $\Delta$ is the unit disk in $\mathbb{C}$ and $\Delta^{*}=\Delta \backslash\{0\}$.

The quasi-coordinate maps can be constructed as follows: Let

$$
\Delta_{3 / 4}=\{v \in \mathbb{C} ;|v|<3 / 4\},
$$


and define a holomorphic map $\phi_{\eta}:\left(\Delta_{3 / 4}\right)^{k} \times \Delta^{n-k} \rightarrow U^{*}$ by

$$
\begin{aligned}
& \phi_{\eta}^{i}(v)=e^{\left(1+\eta^{i}\right) /\left(1-\eta^{i}\right) \cdot\left(v^{i}+1\right) /\left(v^{i}-1\right)}, \quad i=1, \ldots, k, \\
& \phi_{\eta}^{j}(v)=v^{j}, \quad j=k+1, \ldots, n,
\end{aligned}
$$

for each $\eta=\left(\eta^{1}, \ldots, \eta^{k}\right) \in(0,1)^{k}$. It follows that $U^{*}$ is covered by the union

$$
\bigcup_{\eta \in(0,1)^{k}} \phi_{\eta}\left(\left(\Delta_{3 / 4}\right)^{k} \times \Delta^{n-k}\right)
$$

The essential point here is the invariance of the Poincaré metrics under the map $\phi_{\eta}$ : Let $z^{i}=\phi_{\eta}^{i}(v)$; the pullback metric

$$
\left(\phi_{\eta}^{i}\right)^{*}\left(\frac{d z^{i} \wedge d \bar{z}^{i}}{\left|z^{i}\right|^{2}\left(\log \left|z^{i}\right|^{2}\right)^{2}}\right)=\frac{d v^{i} \wedge d \bar{v}^{i}}{\left(1-\left|v^{i}\right|^{2}\right)^{2}}, \quad i=1, \ldots, k
$$

which is independent of $\eta$. In particular, all the derivatives of the pullback metric are bounded on $\Delta_{3 / 4}$.

Now let $U$ run through all the neighborhoods. We get a collection of quasi-coordinate maps $\left\{V_{\eta}, \phi_{\eta}\right\}$, in which each $V_{\eta}$ is a ploydisk of the form

$$
\left(\Delta_{3 / 4}\right)^{l} \times \Delta^{n-l}, \quad 0 \leq l \leq n,
$$

such that $M$ is covered by the union of all the $\phi_{\eta}\left(V_{\eta}\right)$ and that the pullback metric $\phi_{\eta}^{*}(\omega)$ on $V_{\eta}$ and all its derivatives are bounded. In this sense, we say $(M, \omega)$ has bounded geometry up to infinite order.

The bounded geometry setting is particularly useful for applying the techniques of partial differential equations into the geometry of the quasiprojective manifolds, since the natural operators associated with the metric, say Laplacian, will be uniformly elliptic on the quasi-coordinate charts. For this purpose, let us define the Cheng-Yau Hölder spaces as follows: For $k \geq 0$ and $\alpha \in(0,1)$, define the norm $\|\cdot\|_{k, \alpha}$ on $C^{\infty}(M)$ by

$$
\|f\|_{k, \alpha}=\sup _{V_{\eta}}\left\{\left\|\phi_{\eta}^{*}(f)\right\|_{C^{k, \alpha}\left(V_{\eta}\right)}\right\}
$$

Then the Cheng-Yau Hölder space $C^{k, \alpha}(M)$ is defined to be the completion of $\left\{f \in C^{\infty}(M) ;\|f\|_{k, \alpha}<\infty\right\}$ with respect to $\|\cdot\|_{k, \alpha}$. It follows that 
$C^{k, \alpha}(M)$ with $\|\cdot\|_{k, \alpha}$ is a Banach space. Let

$$
\mathcal{R}(M)=\bigcap_{k \geq 0,0<\alpha<1} C^{k, \alpha}(M) .
$$

It is a Fréchet space consisting of all the smooth functions on $M$ whose all derivates are bounded with respect to $\omega$. In particular, we have

$$
\sigma_{i}^{-1}=-\frac{1}{\log \left|s_{i}\right|^{2}} \in \mathcal{R}(M),
$$

for each holomorphic defining section $s_{i}$ of $D_{i}, i=1, \ldots, p$. Here each $\sigma_{i}$ can be viewed as a natural distance function with respect to $D_{i}$. Furthermore, $\sigma_{i}$ induces $\sigma_{i}^{-r} C^{k, \alpha}(M)$, the Cheng-Yau Hölder space with weight $r \in \mathbb{R}$, which is the Banach space defined as usual, i.e., $f \in \sigma_{i}^{-r} C^{k, \alpha}(M)$ if and only if

$$
\sigma_{i}^{r} f \in C^{k, \alpha}(M)
$$

Notice that the weighted Cheng-Yau Hölder spaces are independent of the choice of metrics on each $\left[D_{i}\right]$.

The positive weighted spaces have been used in [26] to characterize the bounded solution of a nondegenerate Monge-Ampère equation. In the situation here, we are going to handle the degenerate case, which naturally has unbounded solutions. So it is necessary to extend to the negative weighted Cheng-Yau spaces. In this paper, we only need the case of weight -1 ; namely, $\sigma_{i} C^{k, \alpha}(M)$ for each $i=1, \ldots, p$. Similar to [26], each negative weighted space has a log-filtration:

$$
\sigma_{i} C^{k, \alpha}(M) \supset C^{k, \alpha}(M) \supset \sigma_{i}^{-1} C^{k, \alpha}(M) \supset \cdots
$$

And similarly, we will prove in Section 5 that the Laplacian operator minus one is a linear isomorphism which preserves the log-filtration. In the following analysis, the sum

$$
\sum_{i=1}^{p} \sigma_{i} C^{k, \alpha}(M)
$$

will be considered as an $\mathbb{R}$-vector space rather than a $C^{k, \alpha}(M)$-module. It induces a direct sum modulo $C^{k, \alpha}(M)$, since for $f_{i} \in C^{k, \alpha}(M), i=1, \ldots, p$, 
we have

$$
\sum_{i=1}^{p} f_{i} \sigma_{i} \in C^{k, \alpha}(M)
$$

if and only if

$$
f_{i} \sigma_{i} \in C^{k, \alpha}(M) \text { for all } i=1, \ldots, p .
$$

This simple observation will be used in the proofs of Lemmas 5.1 and 5.2.

Finally, in order to set up a Monge-Ampère-type equation, let us compute the error term which measures the failure of $\omega$ to be Kähler-Einstein. By direct calculation,

$$
\frac{\omega^{n}}{\Omega}=\prod_{i=1}^{p}\left|s_{i}\right|^{-2 a_{i}} e^{-F}
$$

where $a_{i}=\alpha_{i}+1>0$ for $i=1, \ldots, p$, and $F \in \mathcal{R}(M)$. In other words,

$$
\log \left(\frac{\omega^{n}}{\Omega}\right) \in \sum_{i=1}^{p} a_{i} \sigma_{i}+\mathcal{R}(M) \subset \sum_{i=1}^{p} \sigma_{i} \mathcal{R}(M)
$$

Thus, to prove the existence of a Kähler-Einstein metric on $M$ of negative Ricci curvature, it suffices to solve the following Monge-Ampère equation with degenerate right-hand side:

$$
\begin{gathered}
\frac{\left(\omega+d d^{c} u\right)^{n}}{\omega^{n}}=e^{u} \prod_{i=1}^{p}\left|s_{i}\right|^{2 a_{i}} e^{F}, \\
\omega+d d^{c} u>0 \text { on } M .
\end{gathered}
$$

Indeed, if $u \in C^{\infty}(M)$ is a solution, then

$$
\begin{aligned}
\operatorname{Ric}\left(\left(\omega+d d^{c} u\right)^{n}\right) & =\operatorname{Ric}\left(\omega^{n}\right)+\sum_{i=1}^{p} a_{i} d d^{c} \log \left|s_{i}\right|^{2}+d d^{c} F+d d^{c} u \\
& =\omega+d d^{c} u
\end{aligned}
$$

and hence $\omega+d d^{c} u$ defines a Kähler-Einstein metric on $M$ of negative Ricci curvature.

\section{Yau's upper bound lemma}

In this section, we reformulate, respectively, the special cases of Yau's upper bound lemma and Yau's generalized maximum principle, in the setting of 
quasi-projective manifolds. In such setting, we can prove the maximum principle by using a natural global distance function associated with the divisor, rather than the usual distance function of a Riemannian metric. The global distance function is independent of the Levi-Civita connection, and hence works for certain complete Hermitian metrics.

As applications, we prove the uniqueness of the degenerate MongeAmpère equation, and finiteness of the volume of any Kähler-Einstein metric of negative curvature on $M$. The latter is well known.

Let $(M, \omega)$ be the quasi-projective manifold defined in the previous section. Let $\widetilde{\omega}$ be any Hermitian metric on $M$ satisfying the uniform equivalence condition

$$
C^{-1} \omega<\widetilde{\omega}<C \omega
$$

where $C>1$ is a constant. Denote by $\Delta_{\widetilde{\omega}}$ the negative Hermitian Laplacian associated with $\widetilde{\omega}$, i.e.,

$$
\Delta_{\widetilde{\omega}} v=\frac{n \widetilde{\omega}^{n-1} \wedge d d^{c} v}{\widetilde{\omega}^{n}}
$$

for any $v \in C^{2}(M)$.

The following lemma is a special case of Yau's generalized maximum principle (see, for example, [6, p. 340; 27, p. 206] for a general version). The idea is to compare the function with the global distance function $\sum \log \left(-\log \left|s_{i}\right|^{2}\right)$ associated with $D$.

Lemma 3.1. Let $(M, \widetilde{\omega})$ be given as above. For any function $v \in C^{2}(M)$ with

$$
\sup _{M} v<+\infty
$$

there exists a sequence $\left\{x_{k}\right\}$ in $M$ such that

$$
\begin{aligned}
& \lim _{k \rightarrow+\infty} v\left(x_{k}\right)=\sup _{M} v, \\
& \left.\lim _{\substack{k \rightarrow+\infty \\
\limsup _{k \rightarrow+\infty}}} \Delta_{\widetilde{\omega}} v\left(x_{k}\right)\right|_{\widetilde{\omega}}=0,
\end{aligned}
$$

where $|\cdot|_{\widetilde{\omega}}$ is the metric norm associated with $\widetilde{\omega}$. 
Proof. For each $\epsilon>0$, let

$$
v_{\epsilon}=v-\epsilon \log \left(-\prod_{i=1}^{p} \log \left|s_{i}\right|^{2}\right) .
$$

Then $v_{\epsilon} \in C^{2}(M)$ has an upper bound. Indeed, since

$$
\log \left(-\prod_{i=1}^{p} \log \left|s_{i}\right|^{2}\right)(x) \rightarrow+\infty
$$

as $x$ approaches the boundary divisor $D, \sup _{M} v_{\epsilon}$ must be achieved at the interior of $M$, say $x_{\epsilon}$. Then by applying the usual maximum principle, we get

$$
\begin{gathered}
v_{\epsilon}\left(x_{\epsilon}\right) \geq v(x)-\epsilon \log \left(-\prod_{i=1}^{p} \log \left|s_{i}\right|^{2}\right)(x) \\
d v_{\epsilon}\left(x_{\epsilon}\right)=d v\left(x_{\epsilon}\right)-\epsilon d \log \left(-\prod_{i=1}^{p} \log \left|s_{i}\right|^{2}\right)\left(x_{\epsilon}\right)=0 \\
d d^{c} v_{\epsilon}\left(x_{\epsilon}\right)=d d^{c} v\left(x_{\epsilon}\right)-\epsilon d d^{c} \log \left(-\prod_{i=1}^{p} \log \left|s_{i}\right|^{2}\right)\left(x_{\epsilon}\right) \leq 0 .
\end{gathered}
$$

Therefore, we have

$$
\begin{gathered}
v\left(x_{\epsilon}\right) \geq v(x)-\epsilon \log \left(-\prod_{i=1}^{p} \log \left|s_{i}\right|^{2}\right)(x), \\
\left|d v\left(x_{\epsilon}\right)\right|_{\widetilde{\omega}} \leq \epsilon C\left|d \log \left(-\prod_{i=1}^{p} \log \left|s_{i}\right|^{2}\right)\left(x_{\epsilon}\right)\right|_{\omega} \leq \epsilon C_{1}, \\
\Delta_{\widetilde{\omega}} v\left(x_{\epsilon}\right) \leq \epsilon C \Delta_{\omega} \log \left(-\prod_{i=1}^{p} \log \left|s_{i}\right|^{2}\right)\left(x_{\epsilon}\right) \leq \epsilon C_{1},
\end{gathered}
$$

in which $C_{1}>0$ is a constant independent of $\epsilon$ and $v$. Hence, letting $\epsilon \rightarrow 0^{+}$, we complete the proof.

With the aid of the above lemma, we can prove the following version of Yau's upper bound lemma (See, for example, [6, Theorem 8, p. 353] for the general case). For completeness, we include here a proof which is due to Yau. 
Lemma 3.2. Let $(M, \widetilde{\omega})$ be given as above. Suppose that a function $u \in$ $C^{2}(M)$ satisfies

$$
\Delta_{\widetilde{\omega}} u \geq f(u)
$$

in which $f \in C^{0}(\mathbb{R})$ with

$$
\liminf _{t \rightarrow+\infty} \frac{f(t)}{t^{\alpha}}>0, \quad \alpha>1 \text { is a constant. }
$$

Then $\sup _{M} u<+\infty$ and $f\left(\sup _{M} u\right) \leq 0$.

Proof. Let us assume that $\sup _{M} u=+\infty$. We want to construct a bounded $C^{2}$-function $v$ on $M$ such that $\inf _{M} v=0$, and that for any sequence $\left\{x_{\epsilon}\right\}$ in $M, v\left(x_{\epsilon}\right) \rightarrow 0^{+}$if and only if $u\left(x_{\epsilon}\right) \rightarrow+\infty$, as $\epsilon \rightarrow 0^{+}$. In fact, let $\chi$ be a smooth cut-off function which equals 1 on $[2,+\infty)$, equals 0 on $(-\infty, 1]$, and belongs to $(0,1)$ on $(1,2)$; then

$$
v \equiv \chi(u) u^{-\beta}+\chi(3-u)= \begin{cases}u^{-\beta} & \text { if } u(x) \geq 2 \\ 1 & \text { if } u(x) \leq 1\end{cases}
$$

with $\beta>0$ to be chosen, satisfies all the requirements.

Applying Yau's maximum principle (Lemma 3.1) to $-v$, we have that for each sufficiently small $\epsilon>0$, there is an $x_{\epsilon}$ in $M$ such that

$$
\begin{aligned}
& v\left(x_{\epsilon}\right)<\inf _{M} v+\epsilon=\epsilon, \\
& \left|\nabla v\left(x_{\epsilon}\right)\right|_{\widetilde{\omega}}<\epsilon \\
& \Delta_{\widetilde{\omega}} v\left(x_{\epsilon}\right)>-\epsilon .
\end{aligned}
$$

Therefore, at each $x_{\epsilon}$, we have

$$
-\epsilon^{2}<v\left(x_{\epsilon}\right) \Delta_{\widetilde{\omega}} v\left(x_{\epsilon}\right) \leq-\beta \frac{f\left(u\left(x_{\epsilon}\right)\right)}{u^{2 \beta+1}}+\frac{\beta+1}{\beta}\left|\nabla v\left(x_{\epsilon}\right)\right|_{\widetilde{\omega}}^{2}
$$

Now we set $\beta=(\alpha-1) / 2$, and let $\epsilon \rightarrow 0^{+}$; this inequality gives a contraction. Hence, we prove that $\sup u<+\infty$. Then by applying Lemma 3.1 to $u$, we conclude that $f(\sup u) \leq 0$.

As the first application, let us prove the uniqueness of the following degenerate Monge-Ampère equation. 
Lemma 3.3. For any (positive) continuous function $G$ on $M$ and any constant $\lambda>0$, there exists at most one $u \in C^{2}(M)$ satisfying

$$
\begin{aligned}
\frac{\left(\widetilde{\omega}+d d^{c} u\right)^{n}}{\widetilde{\omega}^{n}} e^{-\lambda u} & =G, \\
C \omega>\widetilde{\omega}+d d^{c} u & >C^{-1} \omega, \quad C>0 .
\end{aligned}
$$

Proof. Assume that there are two such solutions $u_{1}$ and $u_{2}$. Let $h=u_{2}-u_{1}$, and $\widetilde{\omega}_{1}=\widetilde{\omega}+d d^{c} h$. Then the first equation becomes

$$
\frac{\left(\widetilde{\omega}_{1}+d d^{c} h\right)^{n}}{\widetilde{\omega}_{1}^{n}}=e^{\lambda h} .
$$

By the arithmetic and geometric mean inequality we have

$$
\Delta_{\widetilde{\omega}_{1}} h \geq n e^{\lambda h / n}-n .
$$

Applying Yau's upper bound lemma (Lemma 3.2) yields

$$
\sup h \leq 0 .
$$

Similarly, we have $\inf h \geq 0$. Hence, $h=0$. This implies that $u_{1}=u_{2}$.

Next, let us prove the finite volume of an arbitrary negative Kähler-Einstein metric on $M$.

Lemma 3.4. Suppose that $\omega_{\mathrm{KE}}$ is a negative Kähler-Einstein metric on $M$. Then there exists a constant $C>0$ such that $\omega_{\mathrm{KE}}^{n}<C \omega^{n}$. Hence, $\omega_{\mathrm{KE}}$ has finite volume.

Proof. Notice that $\omega_{\mathrm{KE}}^{n} / \omega^{n}$ is a well-defined smooth positive function on $M$. Let $G \in C^{\infty}(M)$ such that

$$
e^{G}=\frac{\omega_{\mathrm{KE}}^{n}}{\omega^{n}} .
$$

It suffices to $\operatorname{show} \sup G<+\infty$. Note that

$$
\begin{aligned}
\omega_{\mathrm{KE}} & =\operatorname{Ric}\left(\omega_{\mathrm{KE}}^{n}\right)=d d^{c} G+\operatorname{Ric}\left(\omega^{n}\right) \\
& =\omega+d d^{c} G+d d^{c} f,
\end{aligned}
$$


where $f=-\sum_{i=1}^{p}\left(\alpha_{i}+1\right) \log \left|s_{i}\right|^{2}-F$ with $F \in \mathcal{R}(M)$. Applying the arithmetic and geometric mean inequality to (3.1) yields that

$$
\begin{aligned}
n e^{G / n} & \leq n+\Delta_{\omega} G+\Delta_{\omega} f \\
& \leq n+\Delta_{\omega} G+C_{1}
\end{aligned}
$$

in which $C_{1}>0$ is a positive constant. It follows from Yau's upper bound lemma (Lemma 3.2) that

$$
e^{\sup G}<\left(1+C_{1} / n\right)^{n}
$$

This completes the proof.

\section{General existence}

Consider the following Monge-Ampère equation with degenerate right-hand side:

$$
\begin{aligned}
\frac{\left(\omega+d d^{c} u\right)^{n}}{\omega^{n}} e^{-u} & =\prod_{i=1}^{p}\left|s_{i}\right|^{2 a_{i}} e^{F} \\
\omega+d d^{c} u & >0 \quad \text { on } M
\end{aligned}
$$

where all $a_{i}$ are positive real numbers, and $F \in \mathcal{R}(M)$.

In this section, we will solve the equation by the $\epsilon$-approximation method, introduced by Yau in the second part of his paper [28, pp. 364-389]. The idea is to approximate (4.1) by a sequence of Monge-Ampère equations with nondegenerate right-hand side:

$$
\begin{aligned}
\frac{\left(\omega+d d^{c} u_{\epsilon}\right)^{n}}{\omega^{n}} e^{-u_{\epsilon}} & =\prod_{i=1}^{p}\left(\left|s_{i}\right|^{2}+\epsilon\right)^{a_{i}} e^{F} \\
\omega+d d^{c} u_{\epsilon} & >0 \quad \text { on } M .
\end{aligned}
$$

For each $\epsilon$, there exists a unique solution $u_{\epsilon} \in \mathcal{R}(M)$ such that $\omega_{\epsilon} \equiv \omega+$ $d d^{c} u_{\epsilon}$ is uniformly equivalent to $\omega$. This is guaranteed by the continuity method based on bounded geometry. (This can also be viewed as a special case in Section 6 by setting all $a_{i}=0$.) To show the existence of (4.1), it suffices to derive a $C^{2}$-Hölder estimate of $u_{\epsilon}$ independent of $\epsilon$ on an arbitrary 
relative compact subset of $M$. We first derive a uniform $C^{0}$-estimate for $u_{\epsilon}$ : It follows from (4.3) that

$$
\begin{aligned}
e^{-u_{\epsilon}} & =\frac{\left(\omega_{\epsilon}-d d^{c} u_{\epsilon}\right)^{n}}{\omega_{\epsilon}^{n}} e^{F} \prod_{i=1}^{p}\left(\left|s_{i}\right|^{2}+\epsilon\right)^{a_{i}} \leq C_{1} \frac{\left(\omega_{\epsilon}-d d^{c} u_{\epsilon}\right)^{n}}{\omega_{\epsilon}^{n}} \\
& \leq C_{1}\left(\frac{n-\Delta_{\epsilon}^{\prime} u_{\epsilon}}{n}\right)^{n}
\end{aligned}
$$

where $\Delta_{\epsilon}^{\prime}$ is negative Laplacian associated with $\omega+d d^{c} u_{\epsilon}$, and $C_{1}$ is a positive constant depending only on $\sup F$ and $[D]$. Then applying Lemma 3.2 to $\left(-u_{\epsilon}\right)$ yields

$$
e^{\inf u_{\epsilon}}>C_{1} .
$$

Unfortunately, there is no global uniform upper bound for $u_{\epsilon}$ because of the degeneracy of the equation; we will have, however, the following substitute:

$$
\sup _{M}\left(u_{\epsilon}+\sum_{i=1}^{p} a_{i} \log \left|s_{i}\right|^{2}\right)<C_{2},
$$

where $C_{2}$ is a positive constant independent of $\epsilon$. In fact, let

$$
v_{\epsilon}=u_{\epsilon}+\sum_{i=1}^{p} a_{i} \log \left|s_{i}\right|^{2} .
$$

Then (4.3) becomes

$$
\begin{aligned}
e^{v_{\epsilon}} & =\frac{\left(\omega-\sum_{i=1}^{p} a_{i} d d^{c} \log \left|s_{i}\right|^{2}+d d^{c} v_{\epsilon}\right)^{n}}{\omega^{n}} \prod_{i=1}^{p}\left(\frac{\left|s_{i}\right|^{2}}{\left|s_{i}\right|^{2}+\epsilon}\right)^{a_{i}} e^{-F} \\
& \leq \frac{\left(\omega-\sum_{i=1}^{p} a_{i} d d^{c} \log \left|s_{i}\right|^{2}+d d^{c} v_{\epsilon}\right)^{n}}{\omega^{n}} e^{-\inf F} \\
& \leq\left(\frac{n+C_{2}^{\prime}+\Delta_{\omega} v_{\epsilon}}{n}\right)^{n} e^{-\inf F} .
\end{aligned}
$$

Here the constant $C_{2}^{\prime}>0$ depends only on the trace of the curvature form of $[D]$ with respect to $\omega$. Hence, by Lemma 3.2 to $v_{\epsilon}$ we get (4.5).

Second, we would like to derive a second-order estimate for $u_{\epsilon}$ which is uniform on each relative compact subset of $M$. Based on Yau's classic work, 
we have, for sufficiently large $C$,

$$
\begin{aligned}
\prod_{i=1}^{p} \mid & \left.s_{i}\right|^{-2 a_{i}} e^{C u_{\epsilon}} \Delta_{\epsilon}^{\prime}\left(\prod_{i=1}^{p}\left|s_{i}\right|^{2 a_{i}} e^{-C u_{\epsilon}}\left(n+\Delta u_{\epsilon}\right)\right) \\
\geq & \left(n+\Delta u_{\epsilon}\right) \Delta_{\epsilon}^{\prime}\left(\log \left(n+\Delta u_{\epsilon}\right)-C u_{\epsilon}+\sum_{i=1}^{p} a_{i} \log \left|s_{i}\right|^{2}\right) \\
\geq & \Delta_{\epsilon}^{\prime}\left(\Delta u_{\epsilon}\right)-\frac{\left|\nabla_{\epsilon}^{\prime}\left(\Delta u_{\epsilon}\right)\right|^{2}}{n+\Delta u_{\epsilon}}-C n\left(n+\Delta u_{\epsilon}\right) \\
& +\left(C-C_{D}\right)\left(n+\Delta u_{\epsilon}\right) \sum_{k=1}^{n} \frac{1}{1+\left(u_{\epsilon}\right)_{k \bar{k}}} \\
\geq & \left(\sum_{i=1}^{p} a_{i} \Delta \log \left(\left|s_{i}\right|^{2}+\epsilon\right)+\Delta F-n^{2} \inf _{k \neq l} R_{k \bar{k} l \bar{l}}\right)-(C n-1)\left(n+\Delta u_{\epsilon}\right) \\
& +\left(C-C_{D}+\inf _{k \neq l} R_{k \bar{k} l \bar{l}}\right)\left(n+\Delta u_{\epsilon}\right) \sum_{k=1}^{n} \frac{1}{1+\left(u_{\epsilon}\right)_{k \bar{k}}} \\
\geq & -C_{3}-C_{4}\left(n+\Delta u_{\epsilon}\right)+C_{5}\left(\prod_{i=1}^{p}\left|s_{i}\right|^{2 a_{i} /(n-1)}\left(n+\Delta u_{\epsilon}\right)^{n /(n-1)}\right)
\end{aligned}
$$

where $C_{D}, C_{3}, C_{4}$ and $C_{5}$ are positive constants independent of $\epsilon$. Note that $\prod_{i=1}^{p}\left|s_{i}\right|^{2 a_{i}} e^{-C u_{\epsilon}}\left(n+\Delta u_{\epsilon}\right)$ must achieve its maximum at the interior of $M$. Let us denote the maximum point by $x$. Then by applying the usual maximum principle, we have

$$
\prod_{i=1}^{p}\left|s_{i}\right|^{2 a_{i} /(n-1)}\left(n+\Delta u_{\epsilon}\right)^{n /(n-1)}<2 \max \left\{C_{3}, C_{4}\left(n+\Delta u_{\epsilon}\right)\right\}
$$

at $x$. Hence, as $0 \leq\left|s_{i}\right|^{2}<1$ for $i=1, \ldots, p$, we get

$$
\prod_{i=1}^{p}\left|s_{i}\right|^{2 a_{i}}\left(n+\Delta u_{\epsilon}\right)<C_{5} \equiv\left(2 C_{3}\right)^{(n-1) / n}+\left(2 C_{4}\right)^{n-1}
$$


at $x$. This, together with the zero-order estimates (4.4) and (4.5), implies that

$$
\begin{aligned}
0<n+\Delta u_{\epsilon} & \leq C_{5} e^{-C u_{\epsilon}(x)} e^{C u_{\epsilon}} \prod_{i=1}^{p}\left|s_{i}\right|^{-2 a_{i}} \\
& \leq C_{6} \prod_{i=1}^{p}\left|s_{i}\right|^{-2 a_{i}(1+C)}
\end{aligned}
$$

on $M$, where $C_{6}$ is a positive constant independent of $\epsilon$. It follows that there are positive constants $\Lambda$ and $C_{7}$ independent of $\epsilon$ such that

$$
C_{7}^{-1} \prod_{i=1}^{p}\left|s_{i}\right|^{2 \Lambda} \omega \leq \omega+d d^{c} u_{\epsilon} \leq C_{7} \prod_{i=1}^{p}\left|s_{i}\right|^{-2 \Lambda} \omega .
$$

Once the second-order estimate is done, the third- and higher-order derivatives can be localized on relative compact subsets of $M$, by choosing appropriate cut-off functions. Therefore, letting $\epsilon \rightarrow 0$, we can conclude that $\left\{u_{\epsilon}\right\}$ has a subsequence converging to a solution $u$ of (4.1) and (4.2) such that $u$ is smooth on $M$, and satisfies that

$$
C_{7}^{-1} \prod_{i=1}^{p}\left|s_{i}\right|^{2 \Lambda} \omega \leq \omega+d d^{c} u \leq C_{7} \prod_{i=1}^{p}\left|s_{i}\right|^{-2 \Lambda} \omega .
$$

This completes the proof of Theorem 1.1.

\section{Isomorphism and asymptotics}

In this section we would like to show the statement (2) in Theorem 1.2. The main tool is the isomorphism theorem developed in [26]. We need the negative weight spaces to characterize the unbounded solution arising from the degenerate equation. In this case, we extend the isomorphism theorems to the negative weight spaces. The following lemmas are needed in deriving the asymptotics of the solution. Throughout this paper, $\sigma_{i} \equiv-\log \left|s_{i}\right|^{2}$ for each irreducible component $D_{i}$ of $D$.

Let $\widetilde{\omega}$ be a Hermitian metric uniformly equivalent to $\omega$ on $M$, i.e., there exists a constant $C>0$ such that

$$
C^{-1} \omega<\widetilde{\omega}<C \omega .
$$


Let $\Delta_{\widetilde{\omega}}$ be the negative Hermitian Laplacian associated with $\widetilde{\omega}$, i.e.,

$$
\Delta_{\widetilde{\omega}}(v)=\frac{n \widetilde{\omega}^{n-1} \wedge d d^{c} v}{\widetilde{\omega}^{n}} \text { for all } v \in C^{2}(M) .
$$

Lemma 5.1. $\Delta_{\widetilde{\omega}}-1: \sum_{i=1}^{p} \sigma_{i} C^{k+2, \alpha}(M) \rightarrow \sum_{i=1}^{p} \sigma_{i} C^{k, \alpha}(M)$ is a linear isomorphism.

Proof. The proof consists of two steps. The first step is to show that

$$
\Delta_{\widetilde{\omega}}-1: \sigma_{i} C^{k+2, \alpha}(M) \rightarrow \sigma_{i} C^{k, \alpha}(M)
$$

is a linear isomorphism, for all $i=1, \ldots, p$. Let us fix such an $i$. Define the conjugate operator

$$
\begin{aligned}
L_{i}(v) & =\sigma_{i}^{-1}\left(\Delta_{\widetilde{\omega}}-1\right)\left(\sigma_{i} v\right) \\
& =\Delta_{\widetilde{\omega}} v+2\left\langle d v, \sigma_{i}^{-1} d \sigma_{i}\right\rangle_{\widetilde{\omega}}+\left(\sigma_{i}^{-1} \Delta_{\widetilde{\omega}}\left(\sigma_{i}\right)-1\right) v,
\end{aligned}
$$

for all $v \in C^{2}(M)$. Then it suffices to show that $L_{i}: C^{k+2, \alpha}(M) \rightarrow C^{k, \alpha}(M)$ is a linear isomorphism, given the following commutative diagram.

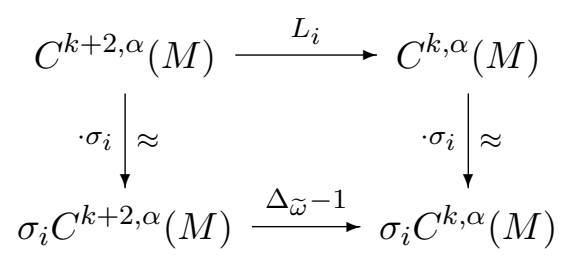

(In this paper, the symbol $\approx$ in all diagrams stands for the linear isomorphism between two vector spaces.) Note that the space $\sigma_{i} C^{k+2, \alpha}(M)$ is independent of the choice of the metric norm $|\cdot|$ for $s_{i} \in H^{0}\left(\bar{M}, \mathcal{O}\left(\left[D_{i}\right]\right)\right)$. We can choose a norm small enough, for instance, by multiplying a small constant, so that

$$
\sigma_{i}^{-1} \Delta_{\widetilde{\omega}} \sigma_{i}<\frac{1}{2}
$$

This is because $\Delta_{\widetilde{\omega}} \sigma_{i} \in \mathcal{R}(M)$ is bounded, and $\sigma_{i}^{-1}$ is small when the supremum of $\left|s_{i}\right|^{2}$ is sufficiently small. Then we claim that

$$
\operatorname{ker} L_{i} \cap C^{k+2, \alpha}(M)=\{0\} .
$$

Indeed, for $v \in C^{k+2, \alpha}(M)$ such that $L_{i} v=0$, applying Yau's generalized maximum principle (Lemma 3.1) yields $\sup v \leq 0$. Similarly, $\sup (-v) \leq 0$, and hence $v=0$. This proves the claim. It follows from the Fredholm 
alternative (see, for example, $\left[26\right.$, p. 811]) that $L_{i}$ is a linear isomorphism from $C^{k+2, \alpha}(M)$ to $C^{k, \alpha}(M)$. This completes the first step.

From the first step we know that

$$
\Delta_{\widetilde{\omega}}-1: \sum_{i=1}^{p} \sigma_{i} C^{k+2, \alpha}(M) \longrightarrow \sum_{i=1}^{p} \sigma_{i} C^{k, \alpha}(M)
$$

is surjective. It remains to prove the injectivity. Suppose that there exist $u_{i} \in C^{k+2, \alpha}(M), i=1, \ldots, p$, such that

$$
\left(\Delta_{\widetilde{\omega}}-1\right)\left(\sum_{i=1}^{p} \sigma_{i} u_{i}\right)=0 .
$$

Equivalently, we have

$$
\sum_{i=1}^{p} \sigma_{i} L_{i}\left(u_{i}\right)=0
$$

This implies that

$$
L_{i}\left(u_{i}\right) \in \sigma_{i}^{-1} C^{k, \alpha}(M) \text { for all } i=1, \ldots, p,
$$

in view of the spaces with negative weight. Then, we have

$$
u_{i} \in \sigma_{i}^{-1} C^{k+2, \alpha}(M) \text { for all } i=1, \ldots, p,
$$

because of the following commutative diagram

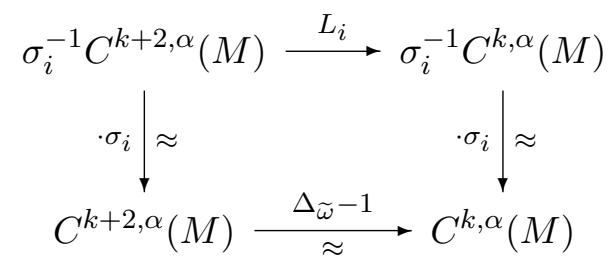

and the injectivity of $L_{i}$ on $C^{k+2, \alpha}(M)$. Hence, $\sum_{i=1}^{p} \sigma_{i} u_{i} \in C^{k+2, \alpha}(M)$. Then, the injectivity of

$$
\Delta_{\widetilde{\omega}}-1: C^{k+2, \alpha}(M) \rightarrow C^{k, \alpha}(M)
$$

forces that $\sum_{i=1}^{p} \sigma_{i} u_{i}=0$. This proves the injectivity of (5.1). Therefore the proof is completed. 
Remark. The proof of Lemma 5.1 implies that $\Delta_{\widetilde{\omega}}-1$ is a linear isomorphism preserving the natural log-filtration of the Cheng-Yau Hölder spaces up to weight $(-1)$. It is possible that the operator has nontrivial kernel on weight $(-2)$ spaces.

In the case of the subspace $\left(\oplus_{i=1}^{p} \mathbb{R} \sigma_{i}\right) \oplus C^{k, \alpha}(M)$, we can say more:

Lemma 5.2. For any constants $b_{i}, i=1, \ldots, p$, the following diagram commutes.

$$
\begin{aligned}
& \sum_{i=1}^{p} b_{i} \sigma_{i}+C^{k+2, \alpha}(M) \underset{\Delta_{\widetilde{\omega}}-1}{\approx}-\sum_{i=1}^{p} b_{i} \sigma_{i}+C^{k, \alpha}(M) \\
& \iota \downarrow \iota \\
& \sum_{i=1}^{p} \sigma_{i} C^{k+2, \alpha}(M) \underset{\Delta_{\widetilde{\omega}}-1}{\approx} \sum_{i=1}^{p} \sigma_{i} C^{k, \alpha}(M)
\end{aligned}
$$

Here the map $\iota$ stands for the inclusion.

Proof. We have only to prove the map on the top is a bijection between the hyperplanes. By the previous lemma, for each $f \in C^{k, \alpha}(M)$, there exist $u_{i} \in C^{k+2, \alpha}(M), i=1, \ldots, p$, such that

$$
\left(\Delta_{\widetilde{\omega}}-1\right)\left(\sum_{i=1}^{p} \sigma_{i} u_{i}\right)=-\sum_{i=1}^{p} b_{i} \sigma_{i}+f .
$$

On the other hand,

$$
\left(\Delta_{\widetilde{\omega}}-1\right)\left(\sum_{i=1}^{p} b_{i} \sigma_{i}\right)=-\sum_{i=1}^{p} b_{i} \sigma_{i}+\sum_{i=1}^{p} b_{i} \Delta_{\widetilde{\omega}}\left(\sigma_{i}\right) .
$$

Hence,

$$
\left(\Delta_{\widetilde{\omega}}-1\right)\left(\sum_{i}\left(u_{i}-b_{i}\right) \sigma_{i}\right)=f-\sum_{i} b_{i} \Delta_{\widetilde{\omega}}\left(\sigma_{i}\right) \in C^{k, \alpha}(M) .
$$

This implies that

$$
\sum_{i=1}^{p} \sigma_{i} L_{i}\left(u_{i}-b_{i}\right) \in C^{k, \alpha}(M)
$$


Hence,

$$
L_{i}\left(u_{i}-b_{i}\right) \in \sigma_{i}^{-1} C^{k, \alpha}(M) \text { for all } i=1, \ldots, p .
$$

It follows that

$$
u_{i}-b_{i} \in \sigma_{i}^{-1} C^{k+2, \alpha}(M) \text { for all } i=1, \ldots, p .
$$

Therefore,

$$
\sum_{i=1}^{p} u_{i} \sigma_{i}-\sum_{i=1}^{p} b_{i} \sigma_{i} \in C^{k+2, \alpha}(M)
$$

This completes the proof.

Now let us start to prove the first part of Theorem 1.2. Assume that there exists a Kähler-Einstein metric $\omega_{\mathrm{KE}}$ of negative Ricci curvature dominating $\omega$. This together with Lemma 3.4 imply that $\omega_{\mathrm{KE}}$ is in fact uniformly equivalent to $\omega$. As in the proof of Lemma 3.4, we let $G \in C^{\infty}(M)$ such that

$$
e^{G}=\frac{\omega_{\mathrm{KE}}^{n}}{\omega^{n}}
$$

Let $u=G-\sum_{i=1}^{p} a_{i} \log \left|s_{i}\right|^{2}-F$ with $a_{i}=\alpha_{i}+1$. We want to show that

$$
u+\sum_{i=1}^{p} a_{i} \log \left|s_{i}\right|^{2} \in \mathcal{R}(M) .
$$

Observe that by $(5.2)$

$$
\begin{aligned}
\omega_{\mathrm{KE}} & =\operatorname{Ric}\left(\omega_{\mathrm{KE}}^{n}\right)=\operatorname{Ric}\left(\omega^{n}\right)+d d^{c} G \\
& =\omega+d d^{c} u .
\end{aligned}
$$

Hence, (5.2) becomes

$$
\frac{\left(\omega+d d^{c} u\right)^{n}}{\omega^{n}}=e^{u} \prod_{i=1}^{p}\left|s_{i}\right|^{2 a_{i}} e^{F} .
$$

Let

$$
\omega_{t}=\omega+t d d^{c} u \text { for all } t \in[0,1] .
$$

In terms of local coordinates, we denote

$$
\omega=\frac{\sqrt{-1}}{2 \pi} \sum_{i, j=1}^{n} g_{i j} d z^{i} \wedge d \bar{z}^{j}, \quad \omega_{t}=\frac{\sqrt{-1}}{2 \pi} \sum_{i, j=1}^{n} g_{t, i \bar{j}} d z^{i} \wedge d \bar{z}^{j} .
$$


It follows that

$$
\begin{aligned}
u+\sum_{i=1}^{p} a_{i} \log \left|s_{i}\right|^{2}+F & =\int_{0}^{1}\left[\frac{d}{d t} \log \operatorname{det}\left(g_{t, i \bar{j}}\right)\right] d t \\
& =\sum_{i, j=1}^{n} A^{i \bar{j}} u_{i \bar{j}}
\end{aligned}
$$

in which

$$
A^{i \bar{j}}=\int_{0}^{1} g_{t}^{i \bar{j}} d t, \quad \sum_{k=1}^{n} g_{t}^{i \bar{k}} g_{t, j \bar{k}}=\delta_{i j}, \quad i, j=1, \ldots, n
$$

Note that $\left\{A^{i \bar{j}}\right\}$ form a tensor on $M$. Let

$$
\widetilde{\omega}=\frac{\sqrt{-1}}{2 \pi} \sum_{i, j=1}^{n} A_{i \bar{j}} d z^{i} \wedge d \bar{z}^{j}
$$

where $A_{i j}$ are obtained by lowering the indexes with respect to the metric $\omega$. In view of the uniform equivalence of $\omega_{\mathrm{KE}}$ and $\omega, \omega_{t}$ is uniformly equivalent to $\omega$. Hence, $\widetilde{\omega}$ defines a Hermitian metric uniformly equivalent to $\omega$ over $M$. We can now rewrite (5.4) as

$$
\left(\Delta_{\widetilde{\omega}}-1\right) u=\sum_{i=1}^{p} a_{i} \log \left|s_{i}\right|^{2}+F
$$

Since $F \in \mathcal{R}(M)$ and $u \in C^{\infty}(M)$, it follows immediately from Lemma 5.2 and the uniqueness (Lemma 3.3) that

$$
u \in-\sum_{i=1}^{p} a_{i} \log \left|s_{i}\right|^{2}+\mathcal{R}(M)
$$

In conclusion, we have derived the following results.

Theorem 5.3. Let $\widetilde{\omega}$ be a Hermitian metric on $M$, which is uniformly equivalent to $\omega$. For any $F \in C^{0}(M)$ and any constants $\lambda>0$ and $b_{i}$, 
$i=1, \ldots, p$, the following equation

$$
\begin{gathered}
\frac{\left(\widetilde{\omega}+d d^{c} u\right)^{n}}{\widetilde{\omega}^{n}}=e^{\lambda u} \prod_{i=1}^{p}\left|s_{i}\right|^{2 b_{i}} e^{F}, \\
C \omega>\widetilde{\omega}+d d^{c} u>C^{-1} \omega, \quad C>1,
\end{gathered}
$$

has at most one solution in $C^{2}(M)$. Furthermore, if such a solution exists, and if $F \in C^{k, \alpha}(M)$ for some $k \geq 0$ and $\alpha \in(0,1)$, then the solution $u$ has the asymptotic form

$$
u+\frac{1}{\lambda} \sum_{i=1}^{p} b_{i} \log \left|s_{i}\right|^{2} \in C^{k+2, \alpha}(M) .
$$

As a consequence, we obtain that

$$
\omega_{\mathrm{KE}}=\omega-\sum_{i=1}^{p} a_{i} d d^{c} \log \left|s_{i}\right|^{2}+d d^{c} v,
$$

for some $v \in \mathcal{R}(M)$; and hence, $\omega_{\mathrm{KE}}$ has bounded sectional curvature and

$$
\omega_{\mathrm{KE}}^{n}=e^{v+F} \omega^{n}=\frac{e^{v} V}{\prod_{i=1}^{p}\left|s_{i}\right|^{2}\left(\log \left|s_{i}\right|^{2}\right)^{2}}
$$

where $V$ is the volume form on $\bar{M}$ (defined in Section 2). This proves the statement (2) in Theorem 1.2.

We remark that the constants $b_{i}$ in Theorem 5.3 need not be positive. The argument of asymptotics work through as long as we have a background metric $\omega$ with bounded curvature and Poincaré growth near $D$. And such a background metric can always be constructed, provided that

$$
K_{\bar{M}}+\sum_{i=1}^{p} \alpha_{i}\left[D_{i}\right]>0 \quad \text { on } \bar{M}
$$

for some real numbers $\alpha_{i}$.

\section{Uniform equivalence}

In this section, we would like to show the first part of Theorem 1.2; namely, if $K_{\bar{M}}+[D]$ restricted to be positive on each irreducible component of $D$, then there exists a Kähler-Einstein metric $\omega_{\mathrm{KE}}$, which is uniformly equivalent 
to $\omega$. By Lemma 6.2 below we can reduce the proof to some estimates near $D$. The key observation is that the following lemma on metric extension can help us to obtain the desired estimates.

Lemma 6.1. Let $X$ be an $n$-dimensional compact complex manifold, and $E=\sum_{i=1}^{q} E_{i}$ be a simple normal crossing divisor in $X$. Let $L$ be a line bundle over $X$ such that $\left.L\right|_{E_{i}}$ is positive for each $i=1, \ldots, q$. Then there exists a smooth metric $h$ of $L$ over $X$ such that the curvature form of $h$ restricted to $E_{i}$ is positive for each $i=1, \ldots, q$.

Remark. It follows from the positivity of $\left.L\right|_{E_{i}}$ that there always exists a metric $h_{i}$ on $L_{E_{i}}$ with positive curvature form on $E_{i}$ for each $i$. The question is whether it is possible to pass the local data $\left\{h_{i}\right\}$ to a global $h$, while preserving the positivity of curvature forms. This is not quite obvious, unless $E$ has only one irreducible component. This lemma is originally proved by Saper [21, p. 212] for complex surfaces; his argument can be straightforwardly generalized to higher dimensions. One can also compare [26, pp. 834-838] for a slightly different setting.

Proof of Lemma 6.1. We first prove that, for a sufficiently large integer $m$, a global section of $m L_{E_{i}}$ can be extended to a global section of $\left.m L\right|_{E}$ for each $i=1, \ldots, q$. It is sufficient to show that, for an arbitrary partial sum, say $\widetilde{E}=\sum_{i=1}^{k-1} E_{i}$, a global section of $\left.m L\right|_{\widetilde{E}}$ can be extended to a global section of $\left.m L\right|_{\widetilde{E}+E_{k}}$. By the short exact sequence

$$
0 \rightarrow \mathcal{O}_{E_{k}}\left(m L-\widetilde{E} \cap E_{k}\right) \rightarrow \mathcal{O}_{\widetilde{E}+E_{k}}(m L) \rightarrow \mathcal{O}_{\widetilde{E}}(m L) \rightarrow 0
$$

we obtain the long exact sequence

$$
H^{0}\left(\widetilde{E}+E_{k}, \mathcal{O}(m L)\right) \rightarrow H^{0}\left(\widetilde{E}, \mathcal{O}_{\widetilde{E}}(m L)\right) \rightarrow H^{1}\left(E_{k}, \mathcal{O}_{E_{k}}\left(m L-\widetilde{E} \cap E_{k}\right)\right)
$$

Note that

$$
H^{1}\left(E_{k}, \mathcal{O}_{E_{k}}\left(m L-\widetilde{E} \cap E_{k}\right)\right)=H^{1}\left(E_{k}, \Omega_{E_{k}}^{n-1}\left(m L-\widetilde{E} \cap E_{k}-K_{E_{k}}\right)\right)=0
$$

for a large $m$, because of $\left.L\right|_{E_{k}}>0$ and applying the Kodaira vanishing theorem. This proves the extension of global sections.

On the other hand, the global sections of $\left.m L\right|_{E}$ define a holomorphic map of $E$ into a large projective space. Hence, by applying the Kodaira embedding theorem and extending the sections, the map restricted to each $E_{i}$ is an embedding, provided that $m$ is sufficiently large. Let us fix such an 
$m$. Since $\left.m L\right|_{E}$ is the pullback of the hyperplane bundle on the projective space, we obtain a metric $h_{E}$ on $\left.m L\right|_{E}$ by pulling back the standard metric on the hyperplane bundle. Then $h_{E}^{1 / m}$ is a metric on $\left.L\right|_{E}$ such that $\left.h_{E}^{1 / m}\right|_{E_{i}}$ is a smooth metric on $\left.L\right|_{E_{i}}$ with positive curvature form for $i=1, \ldots, q$. Finally, patching $h_{E}^{1 / m}$ with any smooth extension gives the desired metric $h$ on $L$.

Let us return to the proof of Theorem 1.2. Let $L=K_{\bar{M}}+[D]$. Note that

$$
\omega_{K}-\sum_{i=1}^{p}\left(\alpha_{i}+1\right) d d^{c} \log h_{i},
$$

represents the first Chern class of $L$, where $h_{i}$ is the metric on $\left[D_{i}\right]$. Let $a_{i}=\alpha_{i}+1$. By the previous lemma, there exists a smooth function $f$ defined on $\bar{M}$ such that

$$
\widetilde{\omega}_{K} \equiv \omega_{K}-\sum_{i=1}^{p} a_{i} d d^{c} \log h_{i}+d d^{c} f
$$

restricted to each $D_{i}$ is positive. Then $\left.\widetilde{\omega}_{K}\right|_{D_{i}}$ is uniformly equivalent to $\left.\omega_{K}\right|_{D_{i}}$, since $\omega_{K}>0$ all over $\bar{M}$ and by the compactness of $D_{i}$. Now comparing $\omega$ with

$$
\widetilde{\omega}_{K}-2 \sum_{i=1}^{p} \sigma_{i}^{-1} \omega_{D_{i}}+2 \sum_{i=1}^{p} \sigma_{i}^{-2} d \sigma_{i} \wedge d^{c} \sigma_{i}=\omega-\sum_{i=1}^{p} a_{i} d d^{c} \log h_{i}+d d^{c} f,
$$

both have the same term $\sum_{i=1}^{p} \sigma_{i}^{-2} d \sigma_{i} \wedge d^{c} \sigma_{i}$, which is dominant near $D$ in the normal direction. Hence, they are uniformly equivalent on a neighborhood of $D$. In particular, the following (6.1) is satisfied. Hence, we complete the proof of Theorem 1.2, with the aid of the following lemma.

Lemma 6.2. Suppose that there exist two positive constants $\delta$ and $\lambda$ such that

$$
\omega-\sum_{i=1}^{p} a_{i} d d^{c} \log \left|s_{i}\right|^{2}+d d^{c} f>\lambda \omega \quad \text { on } D_{\delta} \backslash D
$$

for some bounded function $f \in C^{2}\left(D_{\delta}\right)$, where $D_{\delta^{\prime}} \equiv \cup_{i=1}^{p}\left\{\left|s_{i}\right|<\delta^{\prime}\right\}$ for any $0<\delta^{\prime}<1$. Then there exists a Kähler-Einstein metric $\omega_{\mathrm{KE}}$ which is uniformly equivalent to $\omega$. 
Proof. It is sufficient to solve the following Monge-Ampère equation:

$$
\begin{aligned}
\frac{\left(\omega+d d^{c} u\right)^{n}}{\omega^{n}} & =e^{u} \prod_{i=1}^{p}\left|s_{i}\right|^{2 a_{i}} e^{F}, \\
C^{-1} \omega<\omega+d d^{c} u & <C \omega,
\end{aligned}
$$

where $F \in \mathcal{R}(M)$. Let us fix a $k \geq 3$ and an $\alpha \in(0,1)$ so that $F \in C^{k, \alpha}(M)$.

Let

$$
\mathcal{U}=\left\{u \in C^{2}(M) ; C^{-1} \omega<\omega+d d^{c} u<C \omega \text { for some } C>1 .\right\}
$$

and

$$
\begin{aligned}
T= & \left\{t \in[0,1] ; \frac{\left(\omega+d d^{c} u\right)^{n}}{\omega^{n}}=e^{u} \prod_{i=1}^{p}\left|s_{i}\right|^{2 a_{i} t} e^{t F} \text { has a solution } u \in \mathcal{U}\right. \\
& \text { with } \left.u+t \sum_{i=1}^{p} a_{i} \log \left|s_{i}\right|^{2} \in C^{k+2, \alpha}(M)\right\} .
\end{aligned}
$$

This is the classical form of the continuity method. The aim is to show that $1 \in T$. Here, the nonemptiness of $T$ is trivial, since $0 \in T$. The openness of $T$ follows from the usual implicit function theorem for Banach spaces. Indeed, for $t \in T$, there is a function $v \in C^{k+2, \alpha}(M)$ such that

$$
C^{-1} \omega<\omega+d d^{c} v-t \sum_{i=1}^{p} a_{i} d d^{c} \log \left|s_{i}\right|^{2}<C \omega
$$

for some $C>1$, and that

$$
\mathcal{M}(t, v) \equiv \log \frac{\left(\omega+d d^{c} v-t \sum_{i=1}^{p} a_{i} d d^{c} \log \left|s_{i}\right|^{2}\right)^{n}}{\omega^{n}}-v=t F
$$

Note that $\mathcal{M}$ defines a map from an open subset of $[0,1] \times C^{k+2, \alpha}(M)$ to $C^{k+2, \alpha}(M)$, and the partial Fréchet derivative of $\mathcal{M}$ at $(t, v)$ with respect to $v$ is a linear isomorphism between $C^{k+2, \alpha}(M)$ and $C^{k, \alpha}(M)$, since

$$
D_{2} \mathcal{M}(t, v)=\Delta_{t, v}-1
$$

Here we denote by $\Delta_{t, v}$ the negative Laplacian associated with the metric $\omega+d d^{c} v-t \sum a_{i} d d^{c} \log \left|s_{i}\right|^{2}$. Hence, there is an open neighborhood of $t$ belongs to $T$. This proves the openness. 
For closedness, it is sufficient to establish a uniform $C^{3}$-estimate for

$$
v \equiv u+t \sum_{i=1}^{p} a_{i} \log \left|s_{i}\right|^{2} \in C^{k+2, \alpha}(M), \quad t \in[0,1] .
$$

The $C^{3}$ estimate depends on a uniform $C^{2}$ estimate which, in turn, depends on a uniform $C^{0}$ estimate of $v$.

Note that, as in Section 4, we can always get the global uniform upper bound of $v$ and lower bound of $u$ by applying Yau's upper bound lemma to the Monge-Ampère equation. Rewriting the Monge-Ampère equation in terms of $v$ and applying the arithmetic and geometric mean inequality yields

$$
\begin{aligned}
e^{v} & =\frac{\left(\omega-t \sum_{i=1}^{p} a_{i} d d^{c} \log \left|s_{i}\right|^{2}+d d^{c} v\right)^{n}}{\omega^{n}} e^{-t F} \\
& \leq\left(\frac{n+C_{2}^{\prime}+\Delta_{\omega} v}{n}\right)^{n} e^{|\inf F|}
\end{aligned}
$$

where $C_{2}^{\prime}>0$ depends only on the trace of the curvature form $[D]$ with respect to $\omega$. By Lemma 3.2, we have

$$
e^{\sup v} \leq\left(1+C_{2}^{\prime}\right)^{n} e^{|\inf F|} .
$$

Similarly, we have

$$
\begin{aligned}
e^{-u} & =\frac{\left(\omega_{u}-d d^{c} u\right)^{n}}{\omega_{u}^{n}} e^{t F} \prod_{i=1}^{p}\left|s_{i}\right|^{2 t a_{i}} \\
& \leq\left(\frac{n-\Delta_{\omega_{u}} u}{n}\right)^{n} e^{|\sup F|}
\end{aligned}
$$

where $\omega_{u} \equiv \omega+d d^{c} u \in \mathcal{U}$. Hence, applying Lemma 3.2 yields

$$
e^{\inf u} \geq e^{-|\sup F|}
$$

The hard part is to derive a global uniform lower bound for $v$. It is here that we need the assumption of (6.1). The argument is as follows:

(i) If $v$ achieves its infimum at some point in $\bar{M} \backslash D_{\delta / 2}$, then by (6.3) we have

$$
v=u+t \sum_{i=1}^{p} a_{i} \log \left|s_{i}\right|^{2} \geq-|\sup F|+2 \sum_{i=1}^{p} a_{i} \log (\delta / 2) .
$$


(ii) If not, then we consider $v$ on $D_{\delta} \backslash D$. In this case, $v$ does not take its infimum on the boundary of $D_{\delta}$. It follows from (6.1) that

$$
\begin{aligned}
\omega_{u}-d d^{c}(v-t f) & >[1+t(\lambda-1)] \omega \\
& \geq \lambda \omega \text { for all } t \in[0,1]
\end{aligned}
$$

where we assume $\lambda \in(0,1)$, without loss of generality. Then by the Monge-Ampère equation

$$
\begin{aligned}
e^{-(v-t f)} & =\frac{\omega^{n}}{\omega_{u}^{n}} e^{t F+t f} \\
& \leq \frac{1}{\lambda^{n}}\left(\frac{n-\Delta_{\omega_{u}}(v-t f)}{n}\right)^{n} e^{|\sup F|+|\sup f|}
\end{aligned}
$$

Hence, by the proof of Lemma 3.2 we get

$$
e^{v-t f} \geq \lambda^{n} e^{-|\sup F|-|\sup f|} \quad \text { on } D_{\delta} \backslash D
$$

and hence

$$
v \geq n \log \lambda-|\sup F|-|\sup f|-|\inf f| \quad \text { on } D_{\delta} \backslash D \text {. }
$$

It follows from (i) and (ii) that

$$
\inf _{M} v>-C_{8}
$$

for a positive constant $C_{8}$ independent of $t$.

Next we would like to derive a global uniform second-order estimate for $v$. It is standard to derive

$$
e^{-C u}\left(n+\Delta_{\omega} u\right)<C_{9}
$$

by using the estimates of $\inf u$ and $\sup v$, where $C$ is a sufficiently large constant and $C_{9}$ is a positive constant independent of $t$. (The argument is similar to that in Section 4 by setting all $a_{i}=0$ and $\epsilon=0$, except that at the last step Lemma 3.2 is used instead of the usual maximum principle. See also the estimate below.)

We still need a global uniform estimate for $e^{-C v}(n+\Delta u)$, which depends on (6.1). Again, let us consider two cases: If $e^{-C v}(n+\Delta u)$ achieves its 
supremum at some point in $\bar{M} \backslash D_{\delta / 2}$, then

$$
\begin{aligned}
e^{-C v}(n+\Delta u) & =\prod_{i=1}^{p}\left|s_{i}\right|^{-2 a_{i} C t} e^{-C u}(n+\Delta u) \\
& \leq C_{9}(2 / \delta)^{2 C \sum_{i=1}^{p} a_{i}} .
\end{aligned}
$$

Otherwise, let us consider $e^{-C v}(n+\Delta u)$ in $D_{\delta} \backslash D$. By the local assumption (6.1), using a normal coordinate chart near a point we have

$$
\begin{aligned}
\Delta^{\prime} & \left(e^{-C(v-t f)}(n+\Delta u)\right) \\
\geq & e^{-C(v-t f)}(n+\Delta u)\left(-C \Delta^{\prime}(v-t f)+\Delta^{\prime} \log (n+\Delta u)\right) \\
\geq & e^{-C(v-t f)}(n+\Delta u)\left(-n C+\sum_{k=1}^{n} \frac{C \lambda}{1+u_{k \bar{k}}}\right) \\
& +e^{-C(v-t f)}\left(\Delta^{\prime}(\Delta u)-\frac{\left|\nabla^{\prime}(\Delta u)\right|^{2}}{n+\Delta u}\right) \\
\geq & e^{-C(v-t f)}\left(t \sum_{i=1}^{p} a_{i} \Delta \log \left|s_{i}\right|^{2}+t \Delta F-n^{2} \inf _{k \neq l} R_{k \bar{k} l \bar{l}}-n\right) \\
& -(C n-1) e^{-C(v-t f)}(n+\Delta u) \\
& +\left(C \lambda+\inf _{k \neq l} R_{k \bar{k} l \bar{l}}\right) e^{-C(v-t f)}(n+\Delta u) \sum_{k=1}^{n} \frac{1}{1+u_{k \bar{k}}} \\
\geq & -C_{10}-(n C+1)\left(e^{-C(v-t f)}(n+\Delta u)\right) \\
& +C_{11}\left(e^{-C(v-t f)}(n+\Delta u)\right)^{n /(n-1)},
\end{aligned}
$$

where the constant $C$ is chosen such that $C \lambda+\inf _{k \neq l} R_{k \bar{k} l \bar{l}}>1, C_{10}$ and $C_{11}$ are positive constants independent of $t$, and the last inequality uses (6.2) and (6.5). It follows from Lemma 3.2 that

$$
\sup _{D_{\delta} \backslash D}\left(e^{-C(v-t f)}(n+\Delta u)\right) \leq C_{12},
$$

where $C_{12}>0$ is a constant independent of $t$. Therefore,

$$
e^{-C v}(n+\Delta u) \leq C_{12} e^{-C|\inf f|} \quad \text { on } D_{\delta} .
$$

Hence, in both cases we obtain

$$
e^{-C v}(n+\Delta u)<C_{13},
$$


in which $C_{13}$ is a positive constant independent of $t$. This implies that

$$
0<n+\Delta u \leq C_{13} e^{C \sup v}<C_{14}
$$

in which the constant $C_{14}>0$ is independent of $t$. This, together with (6.5), implies that there is a positive constant $C_{15}$ independent of $t$ such that

$$
C_{15}^{-1} \omega<\omega+d d^{c} u<C_{15} \omega
$$

Therefore, we end up with a global uniform second-order estimate for $u$, and equivalently for $v$. The third-order estimate can be localized; in fact, one can derive a global uniform third-order estimate for $u$ and $v$ over $M$, due to (6.6) (we omit the details here since it is not needed). By Ascoli-Arzelà theorem and (6.6) we complete the proof of closedness of $I$.

\section{Restricted nef and almost completeness}

In this section, we will prove Theorem 1.3. Similar to the previous section, we can reduce the proof to some boundary estimates. Such estimates can be obtained by applying the following extension lemma on the nef case, a corollary of Lemma 6.1 indeed.

Lemma 7.1. Let $X$ be a compact complex manifold, and $E=\sum_{i=1}^{q}$ be a simple normal crossing divisor in $X$. Let $H$ be a positive line bundle over $X$, and $\Theta$ a positive $(1,1)$-form represented $c_{1}(H)$. Suppose that $L$ is a line bundle over $X$ such that $\left.L\right|_{E_{i}}$ is nef for each $i$. Then for any $\varepsilon>0$, there exists a smooth metric $h_{\varepsilon}$ of $L$ over $X$ such that

$$
-\left.d d^{c} \log h_{\varepsilon}\right|_{E_{i}}+\left.\varepsilon \Theta\right|_{E_{i}}>0, \quad i=1, \ldots, q
$$

Proof. Fix an $\varepsilon>0$. Since $\left.L\right|_{E_{i}}$ is nef, $\left.L\right|_{E_{i}}+\left.\varepsilon H\right|_{E_{i}}$ is positive, for each $i$. Then the result follows immediately by applying Lemma 6.1 to the line bundle $L+\varepsilon H$.

To apply this lemma, we set

$$
L=K_{\bar{M}}+[D], \quad H=K_{\bar{M}}-\sum_{i=1}^{p} \alpha_{i}\left[D_{i}\right]
$$

Recall that $\omega_{K}$, defined in Section 2, is a positive $(1,1)$-form representing $c_{1}(H)$. It follows from Lemma 7.1 that for every $\varepsilon>0$, there exists a function 
$f_{\varepsilon} \in C^{\infty}(\bar{M})$ such that

$$
\left.\left(\omega_{K}-\sum_{i=1}^{p}\left(\alpha_{i}+1\right) d d^{c} \log h_{i}+d d^{c} f_{\varepsilon}\right)\right|_{D_{i}}>-\left.\varepsilon \omega_{K}\right|_{D_{i}}
$$

Moreover, recall in Section 2 that the background metric is given by

$$
\omega=\omega_{K}+2 \sum_{i=1}^{p} \sigma_{i}^{-1} \omega_{D_{i}}+2 \sum_{i=1}^{p} \sigma_{i}^{-2} d \sigma_{i} \wedge d^{c} \sigma_{i}
$$

in which, near $D$, the last term on the right is dominant in the normal direction. This observation together with (7.1) imply that there exists a positive number $\delta=\delta(\varepsilon)$ such that

$$
\omega-\sum_{i=1}^{p}\left(\alpha_{i}+1\right) d d^{c} \log h_{i}+d d^{c} f_{\varepsilon}>-\varepsilon \omega
$$

on $D_{\delta} \backslash D$, where $D_{\delta}=\cup_{i=1}^{p}\left\{\left|s_{i}\right|^{2}<\delta\right\}$.

Starting from here, we can prove Theorem 1.3, by the following lemma, which is essentially done by [22, p. 581]. Here we provide more details for the nonemptiness of the continuity method.

Lemma 7.2. Suppose that for each $\varepsilon>0$, there exists a constant $\delta=$ $\delta(\varepsilon)>0$ and a bounded function $f_{\epsilon} \in C^{2}\left(D_{\delta}\right)$ such that

$$
\omega-\sum_{i=1}^{p}\left(\alpha_{i}+1\right) d d^{c} \log h_{i}+d d^{c} f_{\varepsilon}>-\varepsilon \omega
$$

on $D_{\delta} \backslash D$. Then there exists an almost-complete Kähler-Einstein metric on $M$.

Let us recall that a Kähler-Einstein metric $\omega_{E}$ on $M$ is said to be almost complete, if there is a sequence $\left\{\omega_{k}\right\}$ of complete Kähler metrics such that

(i) $\operatorname{Ric}\left(\omega_{k}^{n}\right) \leq t_{k} \omega_{k}$, with $\lim t_{k}=1$;

(ii) $\omega_{k}$ converges to $\omega_{E}$ in $C^{\infty}$ uniformly on every compact subset of $M$. 
Proof. Let

$$
\begin{aligned}
T^{\circ}= & \left\{t \in(1,+\infty) ; \frac{\left(\omega+d d^{c} u\right)^{n}}{\omega^{n}}=e^{t u} \prod_{i=1}^{p}\left|s_{i}\right|^{2 a_{i}} e^{F} \text { has a solution } u \in \mathcal{U}\right. \\
& \text { with } \left.u+\frac{1}{t} \sum_{i=1}^{p} a_{i} \log \left|s_{i}\right|^{2} \in C^{k+2, \alpha}(M) .\right\},
\end{aligned}
$$

where $\mathcal{U}$ is the open set given by

$$
\mathcal{U}=\left\{u \in C^{2}(M) ; C^{-1} \omega<\omega+d d^{c} u<C \omega \text { for some } C>1\right\} .
$$

It is sufficient to show that

$$
T^{\circ}=(1,+\infty)
$$

In fact, by the Monge-Ampère equation, we have for each $t \in T^{\circ}$,

$$
\begin{aligned}
\operatorname{Ric}\left(\left(\omega+d d^{c} u\right)^{n}\right) & =\omega+t d d^{c} u \\
& \leq t\left(\omega+d d^{c} u\right)
\end{aligned}
$$

On the other hand, we can always get the global uniform estimate of

$$
e^{-C u}(n+\Delta u)
$$

by the global uniform estimates $\sup \left(t u+\sum a_{i} \log \left|s_{i}\right|^{2}\right)$ and $\inf u$. Hence, $n+\Delta u$ is uniformly bounded on every compact subset of $M$. Hence, we have a uniform $C^{3}$-estimate of $u$ on every compact subset. Therefore, for any sequence $t_{k}$ in $T^{\circ}$ with $\lim t_{k}=1$, there is a subsequence of $u_{i}$ that satisfies (7.3) and converges uniformly on every compact subset to a solution $u \in C^{\infty}(M)$ of the Monge-Ampère equation at $t=1$. Thus, $\omega+d d^{c} u$ is the desired almost-complete Kähler-Einstein metric on $M$.

Hence, it suffices to show that $T^{\circ}$ is nonempty, and both open and closed in $(1,+\infty)$. The openness and closedness can be proved in the same way as in Section 6, except that the global estimates $\inf \left(u+(1 / t) \sum a_{i} \log \left|s_{i}\right|^{2}\right)$ and $\sup (n+\Delta u)$ depend on $t$ because of (7.2). For instance, let us carry out the estimate for $\inf \left(u+(1 / t) \sum a_{i} \log \left|s_{i}\right|^{2}\right)$. Let

$$
v=t u+\sum_{i=1}^{p} a_{i} \log \left|s_{i}\right|^{2} \in C^{k+2, \alpha}(M) .
$$


It suffices to consider $v$ in $D_{\delta} \backslash D$. Note that (7.2) is equivalent to

$$
\omega_{u}-\frac{1}{t} d d^{c}\left(v-f_{\epsilon}\right)>\left(1-\frac{1+\varepsilon}{t}\right) \omega
$$

where $\omega_{u}=\omega+d d^{c} u$. Since $t>1$, we can choose an $\varepsilon>0$, say

$$
\varepsilon=\frac{t-1}{2}
$$

such that $1-(1+\varepsilon) / t>0$. Then, by the Monge-Ampère equation,

$$
\begin{aligned}
e^{-\left(v-f_{\varepsilon}\right)} & =\frac{\omega^{n}}{\omega_{u}^{n}} e^{F+f_{\epsilon}} \\
& \leq\left(\frac{2 t}{t-1}\right)^{n}\left[1-\frac{1}{n t} \Delta_{\omega_{u}}\left(v-f_{\varepsilon}\right)\right]^{n} e^{\sup \left(F+f_{\epsilon}\right)}
\end{aligned}
$$

Applying Lemma 3.2 yields that

$$
e^{v-f_{\varepsilon}} \geq\left(\frac{t-1}{2 t}\right)^{n} e^{-\sup \left(F+f_{\epsilon}\right)} \quad \text { on } D_{\delta} \backslash D
$$

Namely,

$$
v \geq C_{16}(t) \equiv n \log \left(\frac{t-1}{2 t}\right)-\sup \left(F+f_{\epsilon}\right)+\inf f_{\varepsilon} \quad \text { on } D_{\delta} \backslash D
$$

where $\varepsilon=(t-1) / 2$. Hence,

$$
\inf v \geq \min \left\{2 \sum_{i=1}^{p} \log (\delta / 2)-\sup F, C_{16}(t)\right\}
$$

Similarly, we have

$$
0<n+\Delta u<C_{17}(t)
$$

We remark that the positive constant $C_{17}(t)$ blows up when $t \rightarrow 1^{+}$. Finally, the nonemptiness is settled by the following proposition.

Proposition 7.3. There exists a sufficiently large $t \in T^{\circ}$ so that $T^{\circ} \neq \emptyset$. 
Proof. Let us denote by

$$
\omega_{\gamma, w}=\omega+\gamma d d^{c} w-\gamma\left(\sum_{i=1}^{p} a_{i} d d^{c} \log \left|s_{i}\right|^{2}+d d^{c} F\right),
$$

for all $\gamma \in[0,1]$ and $w \in C^{k+2, \alpha}(M)$. Then the Monge-Ampère equation given in the definition of $T^{\circ}$ is equivalent to

$$
\frac{\omega_{1 / t, v}^{n}}{\omega^{n}}=e^{v}
$$

if we set $v=t u+\sum a_{i} \log \left|s_{i}\right|^{2}+F$. Let us fix some large constant $C>1$. Let

$$
\mathcal{V}_{C}=\left\{(\gamma, w) \in[0,1] \times C^{k+2, \alpha}(M) ; C^{-1} \omega<\omega_{\gamma, w}<C \omega\right\}
$$

and define a $C^{\infty}{ }_{-}$map $\mathcal{M}: \mathcal{V}_{C} \rightarrow C^{k, \alpha}(M)$ by

$$
\mathcal{M}(\gamma, w)=\log \frac{\omega_{\gamma, w}^{n}}{\omega^{n}}-w \quad \text { for all }(\gamma, w) \in \mathcal{V}_{C}
$$

Hence, it suffices to show that there exists a pair $(\gamma, v) \in \mathcal{V}_{C}$ with $\gamma \neq 0$, such that

$$
\mathcal{M}(\gamma, v)=0 .
$$

Note that $\mathcal{M}(0,0)=0$. However, the implicit function theorem does not work directly, since the partial Fréchet derivative of $\mathcal{M}$ with respect to $v$ is degenerate at $\gamma=0$. We overcome this difficulty by using the Newton's iteration method, which is completely elementary without using the curvature property of $\omega$.

For a small $\gamma \neq 0$, we want to construct a sequence $\left\{v_{l}\right\}_{l=0}^{\infty}$ such that $v_{0}=0$,

$$
v_{l+1}=v_{l}-D_{2} \mathcal{M}\left(\gamma, v_{l}\right)^{-1}\left(\mathcal{M}\left(\gamma, v_{l}\right)\right), \quad l=0,1,2, \ldots,
$$

and $\left(\gamma, v_{l}\right) \in \mathcal{V}_{C}$ for all $l$, where $D_{2} \mathcal{M}(\gamma, v)$ is the partial Fréchet derivative of $M$ at $(\gamma, v)$ with respect to $v$. Note that

$$
D_{2} \mathcal{M}(\gamma, v)=\gamma \Delta_{\gamma, v}-1
$$

where $\Delta_{\gamma, v}$ is the negative Laplacian associated with $\omega_{\gamma, v}$. Hence, for any $f \in C^{k, \alpha}(M)$, there exists a unique solution $h \in C^{k+2, \alpha}(M)$ for

$$
D_{2} \mathcal{M}(\gamma, v) h=f
$$


as long as $\gamma \neq 0$ and $(\gamma, v) \in \mathcal{V}_{C}$. Applying Yau's maximum principle (Lemma 3.1) and the interior Hölder estimate yields that

$$
\|h\|_{k+2, \alpha} \leq \gamma^{-1} C_{k, \alpha}\|f\|_{k, \alpha},
$$

where the constant $C_{k, \alpha}$ depends only on $k, \alpha, \operatorname{dim} M$ and the constant $C$ associated with $\mathcal{V}_{C}$.

On the other hand, by Newton's method, we have

$$
\begin{aligned}
v_{l+1}-v_{l}= & -D_{2} \mathcal{M}\left(\gamma, v_{l}\right)^{-1}\left(\mathcal{M}\left(\gamma, v_{l}\right)\right. \\
& \left.-\mathcal{M}\left(\gamma, v_{l-1}\right)-D_{2} \mathcal{M}\left(\gamma, v_{l}\right)\left(v_{l}-v_{l-1}\right)\right) \\
= & -D_{2} \mathcal{M}\left(\gamma, v_{l}\right)^{-1} \\
& \left(\int_{0}^{1}(1-\tau) D_{2}^{2} \mathcal{M}\left(\tau v_{l}+(1-\tau) v_{l-1}\right)\left(v_{l}-v_{l-1}, v_{l}-v_{l-1}\right)\right) d \tau .
\end{aligned}
$$

Here the second-order partial derivative

$$
D_{2}^{2} \mathcal{M}(\gamma, v)(h, f)=\gamma^{2} \sum_{i, j, p, q=1}^{n} g_{\gamma, v}^{i \bar{q}} g_{\gamma, v}^{p \bar{j}} h_{i \bar{j}} f_{p \bar{q}},
$$

for all $h, f \in C^{k+2, \alpha}(M)$ and all $(\gamma, v) \in \mathcal{V}_{C}$, and

$$
\omega_{\gamma, v}=\frac{\sqrt{-1}}{2 \pi} \sum_{i, j=1}^{n}\left(g_{\gamma, v}\right)_{i \bar{j}} d z^{i} \wedge d \bar{z}^{j}, \quad \sum_{k=1}^{n} g_{\gamma, v}^{i \bar{k}}\left(g_{\gamma, v}\right)_{j \bar{k}}=\delta_{i j} .
$$

Observe that for any $\left(\gamma, v_{1}\right),\left(\gamma, v_{2}\right) \in \mathcal{V}_{C}$,

$$
\left(\gamma, \tau v_{1}+(1-\tau) v_{2}\right) \in \mathcal{V}_{C} \quad \text { for all } \tau \in[0,1] .
$$

Thus, if $\left(\gamma, v_{j}\right) \in \mathcal{V}_{C}$ for all $j<l+1$, then by (7.5) we obtain

$$
\begin{aligned}
\left\|v_{l+1}-v_{l}\right\|_{k, \alpha} & \leq \frac{\gamma}{2} C_{k, \alpha} C^{2}\left\|\sum_{i, j, p, q} g^{i \bar{q}} g^{p \bar{j}}\left(v_{l}-v_{l-1}\right)_{i \bar{j}}\left(v_{l}-v_{l-1}\right)_{p \bar{q}}\right\|_{k, \alpha} \\
& \leq \gamma C_{n}\left\|v_{l}-v_{l-1}\right\|_{k+2, \alpha}^{2},
\end{aligned}
$$

where the constant $C_{n}$ depends only on $k, \alpha, \omega$ and the constant $C$ associated with $\mathcal{V}_{C}$. 
Since $(0,0) \in \mathcal{V}_{C}$, there exists a small $\delta_{0}>0$ such that for any $\gamma<2 \delta_{0}$, $w \in C^{k+2, \alpha}(M)$ with $\gamma\|w\|_{2, \alpha}<2 \delta_{0}$, we have $(\gamma, w) \in \mathcal{V}_{C}$. Let $v_{0}=0$. Then

$$
\begin{aligned}
\mathcal{M}\left(\gamma, v_{0}\right) & =\log \frac{\omega_{\gamma, 0}^{n}}{\omega^{n}} \\
& =-\gamma \Delta_{\omega}\left(\sum_{i=1}^{p} a_{i} \log \left|s_{i}\right|^{2}+F\right)+\gamma^{2} \varphi,
\end{aligned}
$$

for some $\varphi \in \mathcal{R}(M)$. Let $v_{1}$ be given by (7.4). In view of (7.5) we have

$$
\begin{aligned}
\left\|v_{1}\right\|_{k+2, \alpha} & =\left\|-D_{2} \mathcal{M}(\gamma, 0)^{-1}(\mathcal{M}(\gamma, 0))\right\|_{k+2, \alpha} \\
& \leq C_{k, \alpha} C_{17},
\end{aligned}
$$

in which the constant $C_{17}$ is independent of $\gamma$. Now we fix a sufficiently small $\gamma$ such that

$$
0<\gamma<\min \left\{\delta_{0}, \frac{\delta_{0}}{2 C_{k, \alpha} C_{17}}, \frac{1}{2 C_{n} C_{k, \alpha} C_{17}}\right\} .
$$

Then

$$
\gamma\left\|v_{1}\right\|_{k+2, \alpha} \leq \min \left\{\frac{\delta_{0}}{2}, \frac{1}{2 C_{n}}\right\} ;
$$

in particular, $\left(\gamma, v_{1}\right) \in \mathcal{V}_{C}$. Let $v_{2}$ be given by (7.4). It follows from (7.6) that

$$
\left\|v_{2}-v_{1}\right\|_{k+2, \alpha} \leq \frac{1}{2}\left\|v_{1}\right\|_{k+2, \alpha}
$$

and

$$
\begin{aligned}
\gamma\left\|v_{2}\right\|_{k+2, \alpha} & \leq \gamma\left\|v_{2}-v_{1}\right\|_{k+2, \alpha}+\gamma\left\|v_{1}\right\|_{k+2, \alpha} \\
& \leq \frac{\delta_{0}}{4}+\frac{\delta_{0}}{2}=\frac{3}{4} \delta_{0} .
\end{aligned}
$$

Hence, $\left(\gamma, v_{2}\right) \in \mathcal{V}_{C}$. By (7.4), we obtain by induction a sequence $\left\{v_{l}\right\}$ satisfying that

$$
\left\|v_{l}-v_{l-1}\right\|_{k+2, \alpha} \leq \frac{1}{2^{l-1}}\left\|v_{1}\right\|_{k+2, \alpha},
$$

and

$$
\gamma\left\|v_{l}\right\|_{k+2, \alpha} \leq \sum_{j=1}^{l} \frac{\delta_{0}}{2^{j}}<\delta_{0},
$$


for all $l \in \mathbb{N}$. In particular, each $\left(\gamma, v_{l}\right) \in \mathcal{V}_{C}$. The sequence converges in $\|\cdot\|_{k+2, \alpha}$ to an element $v_{*}$ in $C^{k+2, \alpha}(M)$, as

$$
\begin{aligned}
\sum_{j=1}^{\infty}\left\|v_{l}-v_{l-1}\right\|_{k+2, \alpha} & \leq \sum_{j=1}^{\infty} \frac{1}{2^{l-1}}\left\|v_{1}\right\|_{k+2, \alpha} \\
& \leq 2\left\|v_{1}\right\|_{k+2, \alpha} .
\end{aligned}
$$

Furthermore, $\left(\gamma, v_{*}\right) \in \mathcal{V}_{C}$ since

$$
\gamma\left\|v_{*}\right\|_{k+2, \alpha} \leq 2 \gamma\left\|v_{1}\right\|_{k+2, \alpha} \leq \delta_{0}
$$

Letting $l \rightarrow \infty$ in $(7.4)$ yields that $\mathcal{M}\left(\gamma, v_{*}\right)=0$. Hence,

$$
\gamma^{-1} \in T^{\circ}
$$

with $u_{1 / \gamma}=-\gamma \sum a_{i} \log \left|s_{i}\right|^{2}+v_{*}-F$. This completes the proof.

\section{Acknowledgments}

First of all, the author would like to thank Professor Shing-Tung Yau. From his lectures and papers the author has learned the upper bound lemma together with other techniques for the Monge-Ampère equations. Part of the work was done when the author was at Stanford University; he would like to thank Professor Rick Schoen for his support and encourage. The author would also thank Professors Fangyang Zheng and Matt Stenzel for discussions and their moral support. Finally, he would like to thank the referees for their careful proof reading and helpful questions.

\section{References}

[1] S. Bando, Einstein Kähler metrics of negative Ricci curvature on open Kähler manifolds, Kähler metrics and moduli spaces, Adv. Studies in Pure Math., (Academic Press, Boston, MA) 18, 1990.

[2] E. Calabi, Isometric families of Kähler structures, The Chern Symposium 1979 (Proc. Internat. Sympos., Berkeley, CA, 1979), Springer, New York, Berlin, 1980, 23-39.

[3] H.-D. Cao, Deformation of Kähler metrics to Kähler-Einstein metrics on compact Kähler manifolds, Invent. Math. 81 (1985), 359-372. 
[4] A. Chau, Convergence of the Kähler-Ricci flow on noncompact Kähler manifolds, J. Differential Geom. 66 (2004), 211-232.

[5] S.-Y. Cheng, On the real and complex Monge-Ampère equation and its geometric applications, Proceedings of the International Congress of Mathematicians, Vols 1, 2 (Warsaw, 1983), 533-539.

[6] S.-Y. Cheng and S.-T. Yau, Differential equations on Riemannian manifolds and their geometric applications, Comm. Pure Appl. Math. 28 (1975), 333-354.

[7] S.-Y. Cheng and S.-T. Yau, On the existence of a complete Kähler metric on non-compact complex manifolds and the regularity of Fefferman's equation, Comm. Pure Appl. Math. 33 (1980), 507-544.

[8] S.-Y. Cheng and S.-T. Yau, Inequality between Chern numbers of singular Kähler surfaces and characterization of orbit space of discrete group of $\mathrm{SU}(2,1)$, Contemp. Math. 49 (1986), 31-43.

[9] S.S. Chern, On holomorphic mappings of Hermitian manifolds of the same dimension, Proc. Symp. Pure Math., 11, American Mathematical Society 1968, 157-170.

[10] C. Fefferman, Monge-Ampère equations, the Bergman kernel, and geometry of pseudoconvex domains, Ann. of Math. (2) 103 (1976), 395-416.

[11] D. Gilbarg and N. Trudinger, Elliptic partial differential equations of second order, Springer, 1998.

[12] R. Harmilton, The inverse function theorem of Nash-Moser, Bull. Amer. Math. Soc., 7 (1982), 65-222.

[13] R. Lazarsfeld, Positivity in algebraic geometry I, Springer, Berlin, 2004.

[14] J. Lee, and R. Melrose, Boundary behaviour of the complex MongeAmpère equation, Acta Math. 148 (1982), 159-192.

[15] K. Liu, X. Sun and S.-T. Yau, Canonical metrics on the moduli space of Riemann surfaces I, J. Diff. Geom. 68 (2004), 571-637.

[16] Y.-C. Lu, Holomorphic mappings of complex manifolds, J. Diff. Geom. 2 (1968), 299-312.

[17] N. Mok and S.-T. Yau, Completeness of the Kähler-Einstein metric on bounded domains and the characterization of domains of holomorphy 
by curvature conditions, Proc. Sympos. Pure Math., 39, American Mathematical Society, Providence, RI, 1983.

[18] N. Mok and J.-Q. Zhong, Compactifying complete Kähler manifolds of finite topological type and bounded curvature, Ann. of Math. 129 (1989), 417-470.

[19] J. Moser, A new technique for the construction of solutions of nonlinear differential equations, Proc. Nat. Acad. Sci. 47 (1961), 1824-1831.

[20] H. Royden, The Ahlfors-Schwarz lemma in several complex variables, Comment. Math. Helvetici, 55 (1980), 547-558.

[21] L. Saper, $L_{2}$-cohomology and intersection homology of certain algebraic varieties with isolated singularities, Invent. Math. 82 (1985), 207-255.

[22] G. Tian and S.-T. Yau, Existence of Kähler-Einstein metrics on complete Kähler manifolds and their applications to algebraic geometry, Proc. Conf. San Diego. Adv. Ser. Math. Phys. 1 (1987) 574-629.

[23] G. Tian and S.-T. Yau, Complete Kähler manifolds with zero Ricci curvature. I, J. Amer. Math. Soc. 3 (1990), 579-609.

[24] H. Tsuji, A characterization of ball quotients with smooth boundary, Duke Math. J. 57(2), (1988), 537-553.

[25] W. Wang, On the smooth compactification of Siegel spaces, J. Diff. Geom. 38 (1993), 351-386.

[26] D. Wu, Higher canonical asymptotics of Kähler-Einstein metrics on quasi-projective manifolds, Commun. Anal. Geom. 14, (4) (2006), 795-845.

[27] S.-T. Yau, Harmonic functions on complete Riemannian manifolds, Comm. Pure. Appl. Math. 28 (1975), 201-228.

[28] S.-T. Yau, On the Ricci curvature of a compact Kähler manifold and the complex Monge-Ampère equation I, Comm. Pure Appl. Math. 31, (1978), 339-411.

[29] S.-T. Yau, Métriques de Kähler-Einstein sur les variétés ouvertes, Première Classe de Chern et courbure de Ricci: Preuve de la conjecture de Calabi, Séminaire Palaiseau, Astérisque (Société Mathématique de France, Paris) 58 1978, 163-167. 
[30] S.-T. Yau, A general Schwarz lemma for Kähler manifolds, Amer. J. Math. 100 (1978), 197-203.

[31] S.-T. Yau, A splitting theorem and algebraic geometric characterization of locally Hermitian symmetric spaces, Commun. Anal. Geom., 1 (3) (1993), 473-486.

[32] S.-K. Yeung, Compactification of complete Kähler manifolds with negative Ricci curvature, Invent. Math. 106 (1991), 13-26.

Department of Mathematics

The Ohio State University

1179 UNIVERSITY DRIVE

NEWARK, OH 43055

E-mail address: dwu@math.ohio-state.edu

ReCeIved May 15, 2007 
